\title{
A Communication-avoiding Implicit-Explicit method for a Free-Surface Ocean model
}

\author{
Christopher Newman ${ }^{\mathrm{a}}$, Geoffrey Womeldorff ${ }^{\mathrm{b}}$, Dana A. Knoll ${ }^{\mathrm{c}}$, Luis Chacón ${ }^{\mathrm{d}}$ \\ ${ }^{a}$ Corresponding author. Fluid Dynamics and Solid Mechanics Group (T-3), Los Alamos \\ National Laboratory, PO Box 1663 MS B216, Los Alamos, NM 87545, United States \\ 1-(505)-606-0647 (cnewman@lanl.gov). \\ ${ }^{b}$ Applied Computer Sciences Group (CCS-7), Los Alamos National Laboratory, Los Alamos, \\ NM 87545, United States (womeld@lanl.gov). \\ ${ }^{c}$ Integrated Design and Assessment Group (XTD-IDA), Los Alamos National Laboratory, \\ Los Alamos, NM 87545, United States (nol@lanl.gov). \\ ${ }^{d}$ Applied Mathematics and Plasma Physics Group (T-5), Los Alamos National Laboratory, \\ Los Alamos, NM 87545, United States (chacon@lanl.gov).
}

\begin{abstract}
We examine a nonlinear elimination method for the free-surface ocean equations based on barotropic-baroclinic decomposition. The two dimensional scalar continuity equation is treated implicitly with a preconditioned Jacobian-free Newton-Krylov method (JFNK). The remaining three dimensional equations are subcycled explicitly within the JFNK residual evaluation with a method known as nonlinear elimination. In this approach, the memory footprint of the underlying Krylov vector is greatly reduced over that required by fully coupled implicit methods. The method is second-order accurate and scales algorithmically, with allowed timesteps much larger than fully explicit methods. Moreover, the hierarchical nature of the algorithm lends itself readily to emerging architectures. In particular, we introduce a communication staging strategy for the three dimensional explicit system that greatly reduces the communication costs of the algorithm and provides a key advantage as communication costs continue to dominate relative to floating point costs in emerging architectures.
\end{abstract}

Keywords: ocean modeling, IMEX method, Jacobian-free Newton-Krylov methods, nonlinear elimination, communication-avoiding algorithm

\section{Introduction}

The long-time behavior of the ocean thermohaline circulation under various forcings is an important scientific question impacting a variety of political decisions. It has been shown that high resolution is necessary for an accurate simulation of ocean circulation [1]. Improved ocean simulations follow from resolution of small-scale features, including mesoscale eddies and topographic features. Eddy-resolving simulations have been carried out in fully coupled 
Community Climate System Model simulations [2] as well as in ocean-only configurations [1].

Scientists are currently performing simulations with semi-implicit [3] and split-explicit methods [4, 5]. Typically, these methods utilize a barotropicbaroclinic splitting that isolates fast (barotropic) and slow (baroclinic) time scales. The faster external gravity waves or barotropic motions are independent of depth, and thus two dimensional, while the slower baroclinic motions are fully three dimensional. For most problems of interest, explicit time discretizations are impractical for these systems, due to short timesteps imposed by the fast waves. Due to numerical stability issues resulting from semi-implicit and split-explicit methods, these tools frequently employ lower-than-desired spatial resolution. Having the ability to perform such simulations with reduced splitting errors, using high-resolution grids, and using second-order accurate time integration methods will be a significant step forward towards predictive ocean simulations with greater fidelity.

Fully-implicit ocean models have been developed in recent years $[6,7,8$, $9,10,11]$. These approaches have been motivated to remove timestep restrictions, enhance numerical accuracy, and by the need for high spatial resolution. One advantage of these methods is that relatively large timesteps can be taken without sacrificing second-order accuracy, since the timestep is determined by accuracy instead of numerical stability constraints. A disadvantage, however, is that fully-implicit methods require a non-linear solution for each timestep. In recent years, there has been considerable advancement in both linear and nonlinear solution methods in ocean modeling [12]. In particular, modern methods based on Jacobian-free Newton-Krylov methods (JFNK) [13] have been recently added to the Parallel Ocean Program (POP) $[8,14,15,16]$ at Los Alamos National Laboratory. The JFNK framework allows tighter coupling of the physics, thus reducing numerical errors and improving stability versus operator splitting techniques. In addition, higher-order implicit time integration schemes can be easily implemented in the JFNK framework.

The key to efficient implementation of JFNK is effective preconditioning. Physics-based preconditioning $[8,13,17,18]$ has been particularly successful. In particular, the work of [8] developed a preconditioner strategy for ocean simulation based on barotropic-baroclinic decomposition. The strategy outlined in [8] effectively preconditions the fully coupled three-dimensional vector system by only inverting a scalar, horizontal, two-dimensional system.

In this study, we propose an implicit/explicit (IMEX) method where the two dimensional scalar continuity equation is treated implicitly with preconditioned JFNK, and the remaining three dimensional equations are driven by the barotropic solution and subcycled explicitly within the JFNK residual evaluation. Thus, the fast barotropic physics are treated implicitly while the slower baroclinic physics are treated explicitly. This approach is known as nonlinear elimination since the baroclinic equations are eliminated from the implicit nonlinear residual. After this nonlinear elimination, the resulting nonlinear system consists only of the two dimensional scalar continuity equation [19, 20]. An advantage of nonlinear elimination is that the Krylov memory requirements 
are greatly reduced over the fully implicit method. The work is motivated by successful implementation of an iterated IMEX method for radiation hydrodynamics problems [21, 22], related work on sea-ice modeling [23] and kinetic plasma simulation [19, 24].

It is important to note that the approach described within this manuscript can be thought of in the context of high order/low order (HOLO) methods. The algorithmic idea of HOLO builds on a well-defined hierarchical description (moments) of widely varying space and time scales. The HOLO approach has been very successful in kinetic plasma simulation [19, 24, 25], neutral partical transport [26, 27], and thermal radiative transfer problems [28, 29]. The HOLO approach is a moment-based scale-bridging algorithm, where the coarse scale (LO) problem is obtained via moment integration and is used to accelerate the fine scale $(\mathrm{HO})$ problem. By taking moments, dimensionality is reduced, thus $\mathrm{HO}$ and LO refer to higher and lower dimensionality, respectively. The HOLO method should not be confused with other methods where order refers to convergence with respect to spatial or temporal discretization. The dimensionality of this LO problem is significantly smaller than the HO problem and, therefore, the LO system is far less expensive to solve. The HOLO approach provides multigrid-like algorithmic acceleration: the LO problem solver relaxes the long wavelength aspects of the solution, while the $\mathrm{HO}$ problem solver relaxes only the short wavelength aspects of the solution. Indeed, our approach is characterized by a LO barotropic system obtained by vertical moment of the HO baroclinic continuity and momentum equations.

In both the traditional split-explicit and semi-implicit approaches, the baroclinic or HO system is advanced explicitly with a long timestep $\left(\Delta t_{\mathrm{HO}} \geq \Delta t_{\mathrm{LO}}\right)$. In the split-explicit approach, the barotropic or LO system is subcycled explicitly within the baroclinic system using timesteps $\Delta t_{\mathrm{LO}}$ necessarily much smaller than the baroclinic timestep, $\Delta t_{\mathrm{HO}}$. The semi-implicit approach treats the barotropic system implicitly within the baroclinic system with $\Delta t_{\mathrm{LO}}=\Delta t_{\mathrm{HO}}$. Our approach differs from the traditional split-explicit and semi-implicit approaches because the barotropic system is advanced implicitly and the baroclinic system is explicitly subcycled within the barotropic system. This allows $\Delta t_{\mathrm{LO}}>\Delta t_{\mathrm{HO}}$ while providing a stable time integration strategy.

Motivated by modern and evolving computer architectures, where communication will be the dominant bottleneck, we have devised and implemented a communication staging strategy designed to minimize communication within our explicit $\mathrm{HO}$ solver by reducing the frequency of halo exchanges per timestep. This strategy is a novel combination of ghosting and scheduling, which allows us to partition temporally timesteps contiguously into blocks, in which interprocess communication is performed only at the start of the block. This is to be contrasted against typical implementations, where inter-process communication is performed at each timestep, or each stage in a multistage integration. Our strategy increases the communication halo width from its conventional value of 1 , based on the stencil requirements, to a value based heuristically on the amount of subcycling required. For explicit methods, as in the case of our HO solver, the required halo communication per timestep can be determined a priori. 
This allows the algorithm to perform a single communication call, with larger communication volume, for the HO system per LO timestep. This technique results in a significant reduction of latency, at the expense of performing additional computations in an increased communication halo. We show specifically in $\S 7$ how this communication staging strategy is particularly advantageous to our IMEX method.

The idea of staged communication is not new. In fact the HYCOM Ocean simulation code [30] utilizes a halo width of 6 which allows communication to be executed once per single Runge-Kutta timestep (recall that Runge-Kutta is a multi-stage integration, where communication is conventionally preformed during every stage). The approach of $[31,32,33]$ communicates enough halo width to perform multiple explicit timesteps. However, the end goal for [31, 32, 33 ] is optimization of cache fill, rather than optimization of network bandwidth to minimize data movement and maximize flops. The most similar approach to ours is that of [34], where halo size is expanded for the explicit time integration of PDEs.

The main contribution of this manuscript is the development and demonstration of a first-of-a-kind, second-order accurate in time, iterated IMEX integration scheme for ocean simulation. This manuscript also presents a solid route to heterogeneous computing with a demonstrated, effective communication staging strategy for the explicit HO system, amenable to modern computer architectures.

The manuscript is organized as follows. Section 2 introduces the background and notation for our free-surface, z-level ocean model. Section 3 introduces the mechanics of the IMEX scheme for free-surface ocean simulation. Section 4 reviews the JFNK method and associated preconditioning. Section 5 discusses details of our preconditioning strategy. Section 6 provides algorithmic results that show second-order accuracy in time and a comparison in terms of computational efficiency with the traditional split-explicit method. Section 7 discusses details of the communication staging strategy. Section 8 provides results on the impact of our communication strategy in terms of weak scaling. Conclusions are provided in $\S 9$.

\section{Free-surface ocean formulation}

We assume a $z$-level ocean model in Cartesian coordinates, in $K$ layers, where $k=1$ corresponds to the top layer [3]. The momentum and continuity equations are given by

$$
\begin{gathered}
\frac{\partial \mathbf{u}_{k}}{\partial t}+L_{1}\left(\mathbf{u}_{k}, \eta\right)+f \mathbf{u}_{k}^{\perp}+G_{1}\left(\varphi_{i, k}, \eta\right)+G_{2}\left(\varphi_{i, k}\right)=0, \quad k=1, \ldots, K, \\
\frac{\partial \eta}{\partial t}+D\left(\mathbf{u}_{1}, \eta\right)=0, \quad k=1, \quad \text { and } \\
\nabla \cdot\left(h_{k}(\eta) \mathbf{u}_{k}\right)+w_{k-1 / 2}-w_{k+1 / 2}=0, \quad k=2, \ldots, K,
\end{gathered}
$$


with

$$
\begin{gathered}
L_{1}\left(\mathbf{u}_{k}, \eta\right)=\left(\mathbf{u}_{k} \cdot \nabla\right) \mathbf{u}_{k}+\frac{w_{k-1 / 2} \mathbf{u}_{k-1 / 2}-w_{k+1 / 2} \mathbf{u}_{k+1 / 2}}{h_{k}(\eta)} \\
G_{1}\left(\varphi_{i, k}, \eta\right)=\frac{1}{\rho_{0}} g \nabla \rho_{1}\left(\varphi_{i, k}\right) \eta \\
G_{2}\left(\varphi_{i, k}\right)=\frac{1}{\rho_{0}} \nabla p_{H k}\left(\varphi_{i, k}\right) \text { and } \\
D\left(\mathbf{u}_{1}, \eta\right)=\nabla \cdot\left(h_{1}(\eta) \mathbf{u}_{1}\right)-w_{3 / 2}
\end{gathered}
$$

where the vertical discretization consists of equally spaced layers of thickness $\Delta z$, and $\mathbf{u}_{k}=\left[\begin{array}{ll}u_{k} & v_{k}\end{array}\right]^{\mathrm{T}}$ is the horizontal velocity at mid-layer $k$, the horizontal velocity at the top and bottom of layer $k$ are given by $\mathbf{u}_{k-1 / 2}$ and $\mathbf{u}_{k+1 / 2}$ with $\mathbf{u}_{k \pm 1 / 2}=\left(\mathbf{u}_{k}+\mathbf{u}_{k \pm 1}\right) / 2$. The pressure at the bottom of layer $k$ is given by $p_{k}$, $w_{k-1 / 2}$ is the vertical velocity at the top of layer $k, w_{k}$ is the vertical velocity at mid-layer $k, \eta$ is sea surface height perturbation, $h_{k}$ is the thickness of layer $k$ with $h_{k}=\Delta z_{k}$ for $k \neq 1$ and $h_{1}=\Delta z_{1}+\eta, f$ is the Coriolis parameter, $\mathbf{u}_{k}^{\perp}=\left[\begin{array}{ll}-v_{k} & u_{k}\end{array}\right]^{\mathrm{T}}, \rho_{0}=1000$ is the reference density, $t$ corresponds to time, and $\nabla$ and $\nabla \cdot$ are the gradient and divergence operators in the horizontal plane. We augment (1)-(3) with transport equations for temperature $\varphi_{1, k}$, and salinity $\varphi_{2, k}$ in each layer $k$ :

$$
\frac{\partial}{\partial t}\left(h_{k}(\eta) \varphi_{i, k}\right)+L_{2}\left(\varphi_{i, k}, \mathbf{u}_{k}, \eta\right)=0, \quad i=1,2
$$

with

$$
\begin{aligned}
L_{2}\left(\varphi_{i, k}, \mathbf{u}_{k}, \eta\right)= & \nabla \cdot\left(h_{k}(\eta) \varphi_{i, k} \mathbf{u}_{k}\right) \\
& +\frac{w_{k-1 / 2} h_{k-1 / 2}(\eta) \varphi_{i, k-1 / 2}-w_{k+1 / 2} h_{k+1 / 2}(\eta) \varphi_{i, k+1 / 2}}{h_{k}(\eta)},
\end{aligned}
$$

and a linear equation of state for density given by

$$
\rho_{k}\left(\varphi_{1, k}, \varphi_{2, k}\right)=\rho_{0}\left(1.0-\alpha \varphi_{1, k}+\beta \varphi_{2, k}\right),
$$

with $\alpha=2.5 \times 10^{-4}$ and $\beta=7.6 \times 10^{-4}[15]$. The pressure at the bottom of layer $k$ is given by

$$
\begin{aligned}
p_{k} & =g \rho_{1} \eta+\sum_{l=1}^{k} g \rho_{l} h_{l}, \\
& =g \rho_{1} \eta+p_{H k},
\end{aligned}
$$

where $g$ is acceleration due to gravity and $p_{H k}$ is hydrostatic pressure at the bottom of layer $k$. Due to $(10), p_{k}=p_{k}\left(\varphi_{i, k}\right)$. Unless otherwise stated, we use the following units: $\mathbf{u}_{k}(\mathrm{~m} / \mathrm{s}), w_{k}(\mathrm{~m} / \mathrm{s}), p_{k}\left(\mathrm{~N} / \mathrm{m}^{2}\right), h_{k}(\mathrm{~m}), \eta(\mathrm{m}), f\left(\mathrm{~s}^{-1}\right)$, $\rho_{k}\left(\mathrm{~kg} / \mathrm{m}^{3}\right), t(\mathrm{~s}), \varphi_{1, k}\left({ }^{\circ} \mathrm{C}\right), \varphi_{2, k}(\mathrm{PSU}), x(\mathrm{~m}), y(\mathrm{~m})$ and $z(\mathrm{~m})$. 
For our examples the domain consists of an infinite channel with constant depth, $D$, and length, $L$, with $0 \leq x \leq L,-\infty<y<\infty$ and $-D \leq z \leq 0$ (vertical coordinate, $z$, positive upward; depth, $D$, positive downward). In this configuration we have $\partial(\cdot) / \partial y=0$, and the following boundary conditions:

$$
u_{k}(x=0, y)=u_{k}(x=L, y)=0, k=1, \ldots, K, \quad \text { and } \quad w_{0}=w_{K}=0 .
$$

The fluid is initially at rest. In the remainder of the text we omit the use of subscripts for vertical level and tracer indexing when the meaning is clear.

\section{Implicit/explicit (IMEX) formulation}

We introduce the notation

$$
\overline{\mathbf{u}}=\frac{1}{H(\eta)} \sum_{k=1}^{K} h_{k}(\eta) \mathbf{u}_{k},
$$

where $H(\eta)=\sum_{k=1}^{K} h_{k}(\eta)$ and $\overline{\mathbf{u}}$ is the barotropic velocity obtained by the vertical average of the velocity. Application of barotropic operator (14) to (1)(3) yields the LO problem:

$$
\frac{\partial \eta}{\partial t}+\nabla \cdot H(\eta) \overline{\mathbf{u}}=0
$$

and the HO problem is given by (1)-(3) and (8). Equation (15) is discretized in time using trapezoidal rule [35] with timestep $\Delta t_{\mathrm{LO}}$,

$$
F(\eta):=\eta-\eta^{n}+\frac{\Delta t_{\mathrm{LO}}}{2} \nabla \cdot\left(H(\eta) \overline{\mathbf{u}}(\eta)+H\left(\eta^{n}\right) \overline{\mathbf{u}}^{n}\right)=0,
$$

where $F(\eta)=0$ is solved by a preconditioned JFNK method each timestep. The superscripts indicate LO timestep index. In order to evaluate (16), we need to obtain the term $H(\eta) \overline{\mathbf{u}}(\eta)$ from the HO problem (1)-(3). The HO problem (1)(3) is discretized in time via subcycling with a predictor corrector method. Consider $\eta$ as an approximation to $\eta^{n+1}$ and subcycle $m=0, \ldots, M_{\mathrm{HO}}-1$ steps such that $\Delta t_{\mathrm{LO}}=M_{\mathrm{HO}} \Delta t_{\mathrm{HO}}$ with $M_{\mathrm{HO}}$ chosen such that $\Delta t_{\mathrm{HO}}$ is on order of the dynamical timescale, $\Delta t_{d}$ (defined in $\left.\S 5\right)$ :

$$
\begin{gathered}
\mathbf{u}_{*}-\mathbf{u}_{m}+\Delta t_{\mathrm{HO}}\left(L_{1}\left(\mathbf{u}_{m}, \eta_{m}\right)+f \mathbf{u}_{m}^{\perp}+G_{1}\left(\varphi_{m}, \eta_{m}\right)+G_{2}\left(\varphi_{m}\right)\right)=0, \\
h\left(\eta_{m+1}\right) \varphi_{*}-h\left(\eta_{m}\right) \varphi_{m}+\Delta t_{\mathrm{HO}} L_{2}\left(\varphi_{m}, \mathbf{u}_{m}, \eta_{m}\right)=0, \\
\mathbf{u}_{m+1}-\mathbf{u}_{m}+\frac{\Delta t_{\mathrm{HO}}}{2}\left(L_{1}\left(\mathbf{u}_{m}, \eta_{m}\right)+f \mathbf{u}_{m}^{\perp}+G_{1}\left(\varphi_{m}, \eta_{m}\right)+G_{2}\left(\varphi_{m}\right)\right. \\
\left.+L_{1}\left(\mathbf{u}_{*}, \eta_{m+1}\right)+f \mathbf{u}_{*}^{\perp}+G_{1}\left(\varphi_{*}, \eta_{m+1}\right)+G_{2}\left(\varphi_{*}\right)\right)=0,
\end{gathered}
$$




$$
\begin{aligned}
h\left(\eta_{m+1}\right) \varphi_{m+1}- & h\left(\eta_{m}\right) \varphi_{m} \\
& +\frac{\Delta t_{\mathrm{HO}}}{2}\left(L_{2}\left(\varphi_{m}, \mathbf{u}_{m}, \eta_{m}\right)+L_{2}\left(\varphi_{*}, \mathbf{u}_{m+1}, \eta_{m+1}\right)\right)=0,
\end{aligned}
$$

with $\eta_{m+1}=\alpha_{m} \eta+\left(1-\alpha_{m}\right) \eta^{n}, \eta_{0}=\eta^{n}, \mathbf{u}_{0}=\mathbf{u}^{n}, \varphi_{0}=\varphi^{n}$ and

$$
\alpha_{m}=(m+1) / M_{\mathrm{HO}} .
$$

We note the HO equations (17)-(20) depend on layer $k$, which we have ommitted, and the use of subscripts $m$ for HO (subcycled) timestep indexing. Upon completion of the subcycling process, we have $\overline{\mathbf{u}}(\eta)=\overline{\mathbf{u}}_{M_{\mathrm{HO}} \text {, and the term }}$ $H(\eta) \overline{\mathbf{u}}(\eta)$ in (16) is given by

$$
H(\eta) \overline{\mathbf{u}}(\eta)=\sum_{k=1}^{K} h_{k}\left(\eta_{M_{\mathrm{HO}}}\right) \mathbf{u}_{M_{\mathrm{HO}} k} .
$$

Note that (16)-(20) embody a nonlinear elimination process, as $\mathbf{u}_{m+1}$ and $\varphi_{m+1}$ are obtained as functions of $\eta$ only and thus eliminated from the implicit nonlinear system. The resulting nonlinear system consists only of (16). The subcycling process is implemented inside the JFNK function evaluation (16). Upon convergence of the nonlinear solver, we have a completed LO timestep with $\mathbf{u}^{n+1}=\mathbf{u}_{M_{\mathrm{HO}}}$ and $\varphi^{n+1}=\varphi_{M_{\mathrm{HO}}}$. We note again that the subcycling process is executed for every linear iteration, and thus effective preconditioning is a vital requirement to minimize the total number of function evaluations per LO timestep.

The split-explicit (SE) algorithm is the current method used in the ocean community, e.g. in MPAS [36] and MOM [37]. We provide details of the SE implementation used in this study in $\S$ Appendix A. In SE, the baroclinic HO system is advanced with a long timestep, $\Delta t_{\mathrm{HO}}$, and the barotropic LO system is subcycled with short timesteps, $\Delta t_{\mathrm{LO}}$, restricted by a barotropic CFL limit, within a single $\mathrm{HO}$ timestep. Hence $\Delta t_{\mathrm{LO}}<\Delta t_{\mathrm{HO}}$ necessarily. In practice, the HO and LO systems are coupled via a Picard iteration in order to achieve second-order temporal accuracy [36]. The IMEX formulation has a distinct advantage over the SE method: since the LO system is advanced implicitly and the HO system is subcycled explicitly within the LO system, $\Delta t_{\mathrm{LO}}>\Delta t_{\mathrm{HO}}$, which allows a route to a communication-avoiding algorithm described in $§ 7.2$.

\section{Jacobian-free Newton-Krylov methods}

We briefly describe the JFNK method. More detailed analyses can be found in $[13,38]$. The JFNK method for the discrete problem is

$$
\mathbf{F}(\mathbf{x})=\mathbf{0}
$$

with $\mathbf{F}: \mathbb{R}^{N} \rightarrow \mathbb{R}^{N}$, where $N$ is the number of unknowns. Given the Jacobian, $\mathbf{F}^{\prime}(\mathbf{x})$, it is straightforward to express the Newton iteration,

$$
\mathbf{F}^{\prime}\left(\mathbf{x}^{(i)}\right) \delta \mathbf{x}^{(i)}=-\mathbf{F}\left(\mathbf{x}^{(i)}\right),
$$


and

$$
\mathbf{x}^{(i+1)} \longleftarrow \mathbf{x}^{(i)}+\delta \mathbf{x}^{(i)},
$$

where the superscript $(i)$ denotes the iteration count of the Newton iteration and $\delta \mathbf{x}^{(i)}$ is the Newton step. The Newton iteration is terminated when

$$
\left\|\mathbf{F}\left(\mathbf{x}^{(i)}\right)\right\|_{2}<\tau_{r}\left\|\mathbf{F}\left(\mathbf{x}^{(0)}\right)\right\|_{2}+\tau_{a},
$$

where $\tau_{r}$ is a specified relative tolerance and $\tau_{a}$ is a specified absolute tolerance, and $\|\cdot\|_{2}$ is the Euclidean norm on $\mathbb{R}^{N}$.

Krylov iterative solvers such as the generalized minimum residual (GMRES) algorithm [39] are used to solve the Jacobian system (24), and do not require the Jacobian matrix itself but simply the action of the Jacobian matrix on a vector. Approximating this matrix-vector product by differencing, which requires two nonlinear function evaluations, is the basis of the JFNK method. Specifically, to evaluate the matrix-vector product $\mathbf{F}^{\prime}\left(\mathbf{x}^{(i)}\right) \mathbf{v}$, a finite-difference approach,

$$
\mathbf{F}^{\prime}\left(\mathbf{x}^{(i)}\right) \mathbf{v} \approx \frac{1}{\varepsilon}\left(\mathbf{F}\left(\mathbf{x}^{(i)}+\varepsilon \mathbf{v}\right)-\mathbf{F}\left(\mathbf{x}^{(i)}\right)\right),
$$

is commonly used [13,40], where $\varepsilon$ is chosen heuristically to avoid problems with machine precision [41]. In addition, since $\mathbf{F}\left(\mathbf{x}^{(i)}\right)$ is readily available in the JFNK method, each GMRES iteration requires an evaluation of $\mathbf{F}\left(\mathbf{x}^{(i)}+\varepsilon \mathbf{v}\right)$.

An inexact convergence tolerance is used on the linear solve for the JFNK method [40, 42, 43]. The tolerance to which we solve the linear problem, on each nonlinear iteration, is related to the current nonlinear residual, $\mathbf{F}\left(\mathbf{x}^{(i)}\right)$, by

$$
\left\|\mathbf{F}^{\prime}\left(\mathbf{x}^{(i)}\right) \delta \mathbf{x}^{(i)}+\mathbf{F}\left(\mathbf{x}^{(i)}\right)\right\|_{2} \leq \eta_{(i)}\left\|\mathbf{F}\left(\mathbf{x}^{(i)}\right)\right\|_{2}
$$

with

$$
\eta_{(i)}=\gamma\left(\frac{\left\|\mathbf{F}\left(\mathbf{x}^{(i)}\right)\right\|_{2}}{\left\|\mathbf{F}\left(\mathbf{x}^{(i-1)}\right)\right\|_{2}}\right)^{\alpha}
$$

and

$$
\max \left(\gamma \eta_{(i-1)}^{\alpha}, \eta_{\min }\right) \leq \eta_{(i)} \leq \eta_{\max } .
$$

Our choice of values for $\gamma, \alpha, \eta_{(0)}, \eta_{\min }$ and $\eta_{\max }$ are provided in $\S 6$.

We apply physics-based preconditioning $[44,45]$ as a right preconditioning process $\mathbf{M}^{-1}$ and the matrix vector product expression (27) becomes

$$
\mathbf{F}^{\prime}\left(\mathbf{x}^{(i)}\right) \mathbf{M}^{-1} \mathbf{v} \approx \frac{1}{\varepsilon}\left(\mathbf{F}\left(\mathbf{x}^{(i)}+\varepsilon \mathbf{M}^{-1} \mathbf{v}\right)-\mathbf{F}\left(\mathbf{x}^{(i)}\right)\right) .
$$

The operator $\mathbf{M}^{-1}$ is chosen such that $\mathbf{M}^{-1} \mathbf{v}$ is a suitable approximation to $\mathbf{F}^{\prime}\left(\mathbf{x}^{(i)}\right)^{-1} \mathbf{v}$.

\section{Preconditioner}

We introduce a process for applying the preconditioner. First, we address time scales associated with (16)-(20) that are important for constructing our 
preconditioner. Next, we examine the linearized form of the Jacobian for (16) to arrive at the preconditioner. In contrast to traditional preconditioning, where the Jacobian inverse $\left(\mathbf{F}^{\prime}\right)^{-1}$ is approximated by a matrix $\mathbf{M}^{-1}$ applied to the linear residual vector, we utilize a physics-based preconditioner where the action of $\mathbf{M}^{-1}$ on the linear residual vector $\mathbf{v}$ is approximated by a process. We outline the following process for applying $\mathbf{M}^{-1}$ to a given vector $\mathbf{v}$.

\subsection{Time scales}

We discuss four distinct time scales associated with (16)-(20):

1. External gravity-wave

2. Advection

$$
\Delta t_{g}=\frac{\Delta x}{\sqrt{g D}}=\frac{\Delta x}{c_{f}}
$$

$$
\Delta t_{a}=\frac{\Delta x}{\|\mathbf{u}\|_{2}}=\frac{\Delta x}{c_{s}}
$$

3. Coriolis

$$
\Delta t_{f}=f^{-1}
$$

4. Dynamic

$$
\Delta t_{d}=\left\|\left(\frac{1}{u} \frac{\partial u}{\partial t}\right)^{-1}\right\|_{\infty}
$$

The separation between these time scales presents challenges for time integration. In the deep ocean, the external gravity-wave speed is of order 225 meters per second; the next fastest time scale is associated with advection with speed on order of a few meters per second. Explicit methods are typically unstable for timesteps larger than the external gravity-wave time scale. Typical semiimplicit or split methods utilize an approximate decoupling of (1)-(3) and (8) and treat the physics associated with the external gravity-wave either implicitly, with timesteps on order of the advective time scale; or via explicit subcycling, with many explicit timesteps on order of the external gravity-wave time scale. The physics associated with the advection time scale are treated explicitly with timesteps on order of the advective time scale. The Coriolis physics are typically treated explicitly, although POP treats the Coriolis physics implicitly. The dynamic time scale is inherently problem dependent.

In our approach, (16) is associated with the external gravity-wave timescale, while (17)-(20) are associated with the dynamic time scale. Our goal is to integrate the fully-coupled system (16)-(20) with an implicit time integration for (16) that allows second-order accurate integration with timesteps larger than the external gravity-wave time scale, and on the order of the dynamic time scale. For the example problems considered herein, the Coriolis and advective time scales are much larger than the dynamic time scale. Equations (17)(20) are treated in an explicitly subcycled manner within the JFNK function evaluation using timesteps on order of the dynamic timescale. We will borrow ideas from semi-implicit methods to construct a physics-based preconditioner for the JFNK solver in terms of sea-surface height only, which will allow efficient time integration using timesteps approaching the advection time scale. 


\subsection{Construction of preconditioner}

To construct the preconditioner, we consider the linearization of (16), obtained by directional differentiation of (16) with respect to $\eta$ in a given direction w:

$$
F^{\prime}(\eta) \mathbf{w}=\mathbf{w}+\frac{\Delta t_{\mathrm{LO}}}{2} \nabla \cdot\left(H \overline{\mathbf{u}}^{\prime}(\eta) \mathbf{w}\right),
$$

To find the linearized operator of the subcycling process, $H \overline{\mathbf{u}}^{\prime}(\eta)$ w, we ignore advective terms, terms due to Coriolis and hydrostatic pressure (since they are of slower time scales) and terms involving the constant $\eta^{n}$ in (19). We sum (19) recursively over HO timesteps:

$$
\sum_{m=0}^{M_{\mathrm{HO}}-1} \mathbf{u}^{m+1}-\mathbf{u}^{m}+\frac{\Delta t_{\mathrm{HO}}}{2}\left(G_{1}\left(\varphi^{*}, \eta^{m}\right)+G_{1}\left(\varphi^{m}, \eta^{m+1}\right)\right)=0,
$$

to yield:

$$
\mathbf{u}(\eta) \approx \mathbf{u}^{0}-\frac{\Delta t_{\mathrm{HO}}}{2 \rho_{0}} g \rho_{1} \nabla \eta \sum_{m=0}^{M_{\mathrm{HO}}-1}\left(a_{m}+a_{m+1}\right),
$$

where $a_{m}$ is given in (21), and $\rho_{1}$ is the density in layer $k=1$ obtained from the previous Newton iteration. We use the identity $\sum_{m=0}^{M_{\mathrm{HO}}-1}\left(a_{m}+a_{m+1}\right)=M_{\mathrm{HO}}$ and $M_{\mathrm{HO}} \Delta t_{\mathrm{HO}}=\Delta t_{\mathrm{LO}}$, yielding

$$
\mathbf{u}(\eta)=\mathbf{u}^{0}-g \rho_{1} \frac{\Delta t_{\mathrm{LO}}}{2 \rho_{0}} \nabla \eta .
$$

Linearization and vertical averaging of (39) yields

$$
H \overline{\mathbf{u}}^{\prime}(\eta) \mathbf{w}=-g \rho_{1} \frac{H \Delta t_{\mathrm{LO}}}{2 \rho_{0}} \nabla \mathbf{w},
$$

where we have utilized (14) with $\eta=0$. Substitution of (40) into (36) yields

$$
F^{\prime}(\eta) \mathbf{w}=\left(I-g \rho_{1} \frac{\Delta t_{\mathrm{LO}}^{2}}{4 \rho_{0}} \nabla \cdot H \nabla\right) \mathbf{w} .
$$

Application of the preconditioner, $\mathbf{M}^{-1} \mathbf{v}$, is equivalent to, given $\mathbf{v}$, find $\mathbf{w}$ such that

$$
\left(I-g \rho_{1} \frac{\Delta t_{\mathrm{LO}}^{2}}{4 \rho_{0}} \nabla \cdot H \nabla\right) \mathbf{w}=\mathbf{v} .
$$

The process defined above is equivalent to the preconditioner developed in [8]. In our examples, the system (42) is approximately inverted using multigrid methods [46]. 


\section{Algorithmic results}

We begin this section by estimating the computational cost in approximate flops of SE and IMEX in one horizontal dimension and serial implementation. Cost to integrate SE to some fixed time, $T$ is given by

$$
C_{\mathrm{SE}} \propto 2 N_{\mathrm{P}}\left(\frac{3 T n_{x}}{\Delta t_{\mathrm{LO}}}+\frac{6 T n_{x} K}{\Delta t_{\mathrm{HO}}}\right)
$$

where $N_{\mathrm{P}}$ is the number of Picard iterations per timestep, the common factor of 2 is due to the predictor corrector method, the factor of 3 corresponds to the number of LO variables $(\overline{\mathbf{u}}, \eta)$, the factor of 6 corresponds to the number of HO variables $\left(\mathbf{u}, w, p, \varphi_{i}\right)$ and $n_{x}$ is the number of mesh points in $x$ and $K$ is the number of layers. The first term of (43) represents cost of LO, while the second term represents the cost of HO. From the discussion in 5.1, we define

$$
\begin{aligned}
& \Delta t_{\mathrm{LO}} \sim \frac{\Delta x}{c_{f}} \sim \frac{1}{c_{f} n_{x}}, \\
& \Delta t_{\mathrm{HO}} \sim \frac{\Delta x}{c_{s}} \sim \frac{1}{c_{s} n_{x}},
\end{aligned}
$$

where $c_{f}=\sqrt{g D}$ is a constant associated with the fast external gravity wave (see (32)) and $c_{s}$ is a constant associated with the slower advective timescale with $c_{f} \gg c_{s}$ (see (33)). We can rewrite (43) as

$$
\begin{aligned}
C_{\mathrm{SE}} & \propto 2 N_{\mathrm{P}} T n_{x} K\left(\frac{3}{K \Delta t_{\mathrm{LO}}}+\frac{6}{\Delta t_{\mathrm{HO}}}\right), \\
& \propto 12 N_{\mathrm{P}} T n_{x} K\left(\frac{c_{f} n_{x}}{2 K}+c_{s} n_{x}\right) .
\end{aligned}
$$

For a typical ocean simulation, $n_{x} \gg K$, and the LO term in (47) is bound by the horizontal mesh.

For IMEX, we have

$$
\begin{aligned}
\Delta t_{\mathrm{LO}} & \sim \frac{\nu}{c_{s}}, \\
\Delta t_{\mathrm{HO}} & \sim \frac{1}{c_{s} n_{x}},
\end{aligned}
$$

since the LO component of IMEX is independent of the horizontal mesh, we can pick $\Delta t_{\mathrm{LO}}=\nu c_{s}^{-1}$, where $\nu$ is a positive constant. The cost analysis applied to IMEX yields

$$
\begin{aligned}
C_{\mathrm{IMEX}} & \propto N_{\mathrm{FE}} T n_{x} K\left(\frac{1}{K \Delta t_{\mathrm{LO}}}+\frac{2 \cdot 6}{\Delta t_{\mathrm{HO}}}\right), \\
& \propto 12 N_{\mathrm{FE}} T n_{x} K\left(\frac{c_{s}}{12 \nu K}+c_{s} n_{x}\right),
\end{aligned}
$$

where $N_{\mathrm{FE}}$ is the number of function evaluations per timestep, and the factor of 2 corresponding to the predictor corrector method only appears in the HO 
term and the factor of 6 corresponds to the number of $\mathrm{HO}$ variables. We note that the LO term in (51) is much smaller than the $\mathrm{HO}$ term and that the $\mathrm{HO}$ terms in (47) and (51) are identical. The number of function evaluations per timestep is given by

$$
N_{\mathrm{FE}}=N_{\mathrm{G}}+2 N_{\mathrm{N}}+1,
$$

where $N_{\mathrm{G}}$ is the number of GMRES iterations per timestep, $N_{\mathrm{N}}$ is the number of Newton iterations per timestep, the second term corresponds to an initial function evaluation for GMRES and a function evaluation to check the nonlinear residual each Newton iteration, and the last term corresponds to a function evaluation for the previous timestep as required by trapezoidal rule. The ratio of $C_{\mathrm{IMEX}}$ to $C_{\mathrm{SE}}$ is given by

$$
\begin{aligned}
C_{\mathrm{IMEX}} / C_{\mathrm{SE}} & \approx \frac{N_{\mathrm{FE}}}{N_{\mathrm{P}}}\left(\frac{c_{s}}{12 \nu K}+c_{s} n_{x}\right)\left(\frac{c_{f} n_{x}}{2 K}+c_{s} n_{x}\right)^{-1}, \\
& \approx \frac{N_{\mathrm{FE}}}{N_{\mathrm{P}}}\left(\frac{1}{12 \nu K n_{x}}+1\right)\left(\frac{c_{f}}{2 K c_{s}}+1\right)^{-1}, \\
& \approx \frac{N_{\mathrm{FE}}}{N_{\mathrm{P}}}\left(\frac{c_{f}}{2 K c_{s}}+1\right)^{-1} .
\end{aligned}
$$

In one horizontal dimension we expect (55) to reflect the increase in cost of IMEX over SE, and that the increase in cost is largely dependent on $N_{\mathrm{FE}}$ and $N_{\mathrm{P}}$. Extending the analysis to two horizontal dimensions, we have

$$
\begin{array}{rl}
C_{\mathrm{SE}} & 2 N_{\mathrm{P}} T n_{x} n_{y} K\left(\frac{3 c_{f}\left(n_{x} n_{y}\right)^{1 / 2}}{K}+6 c_{s}\left(n_{x} n_{y}\right)^{1 / 2}\right) \text { and } \\
C_{\mathrm{IMEX}} & \propto 2 N_{\mathrm{FE}} T n_{x} n_{y} K\left(\frac{c_{s}}{2 \nu K}+6 c_{s}\left(n_{x} n_{y}\right)^{1 / 2}\right),
\end{array}
$$

where $n_{y}$ is the number of mesh points in $y$. Again, we see that the HO terms in (56) and (57) are identical, and for IMEX the LO terms in (51) and (57) are also identical. The ratio of $C_{\mathrm{IMEx}}$ to $C_{\mathrm{SE}}$ is given by

$$
\begin{aligned}
C_{\mathrm{IMEX}} / C_{\mathrm{SE}} & \approx \frac{N_{\mathrm{FE}}}{N_{\mathrm{P}}}\left(\frac{c_{s}}{12 \nu K}+c_{s}\left(n_{x} n_{y}\right)^{1 / 2}\right)\left(\frac{c_{f}\left(n_{x} n_{y}\right)^{1 / 2}}{2 K}+c_{s}\left(n_{x} n_{y}\right)^{1 / 2}\right)^{-1} \\
& \approx \frac{N_{\mathrm{FE}}}{N_{\mathrm{P}}}\left(\frac{1}{12 \nu K n_{x}}+1\right)\left(\frac{c_{f}}{2 K c_{s}}+1\right)^{-1} \\
& \approx \frac{N_{\mathrm{FE}}}{N_{\mathrm{P}}}\left(\frac{c_{f}}{2 K c_{s}}+1\right)^{-1}
\end{aligned}
$$

We see that (60) is identical to (55).

We note that for serial IMEX, the dominant cost for integration to a fixed time is in the $\mathrm{HO}$ component. However, for $\mathrm{SE}, \Delta t_{\mathrm{LO}}$ is mesh dependent and the LO cost contributes significantly, particularly in two horizontal dimensions. 


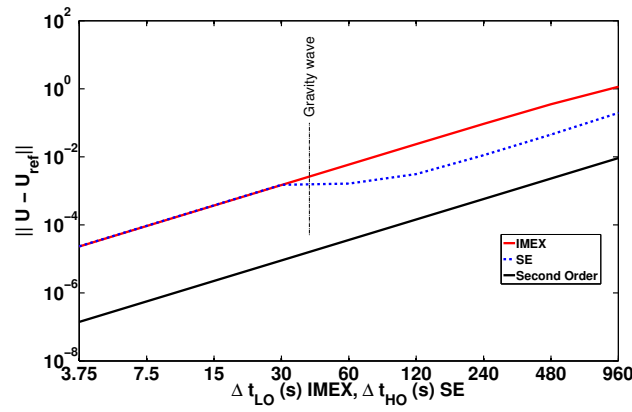

(a)

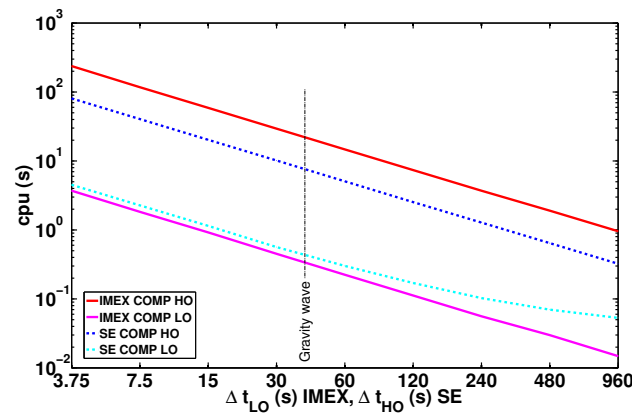

(c)

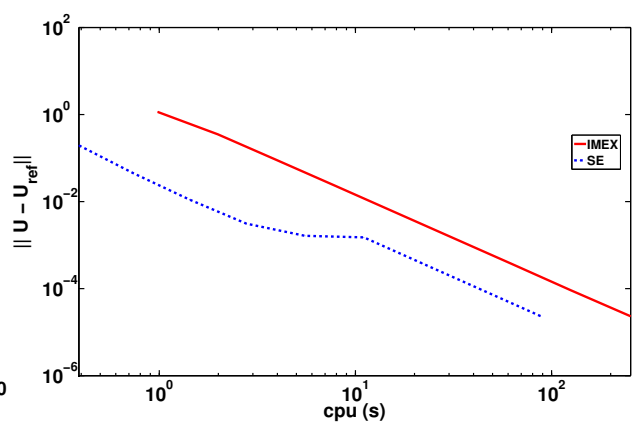

(b)

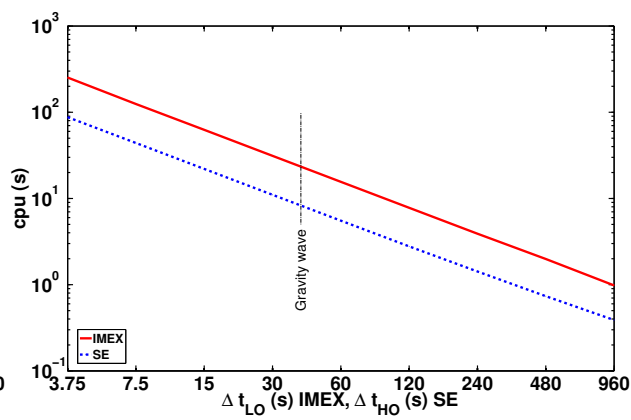

(d)

Figure 1: Convergence and timing results for problem $\S 6.1$. (a) Timestep size vs. error for IMEX and SE. Error rate is computed relative to a reference solution $U_{\text {ref }}$ obtained by fourthorder RK4 with $\Delta t=1.875$. (b) CPU vs. error for IMEX and SE. (c) Timestep size vs. CPU for HO and LO components of IMEX and SE. (d) Timestep size vs. total CPU for IMEX and SE.

In this section, we present two numerical examples to demonstrate convergence of the IMEX method and to compare serial performance of the IMEX method with that of the SE method. In $\S 7.2$, we present a communication strategy that reduces the communication cost particularly for the IMEX method in parallel. The communication strategy is evaluated in parallel for each method in $\S 8$. For each example below, the fluid is initially at rest, with an initial perturbation in $\eta$. The domain consists of a two dimensional infinite channel and is discretized horizontally with a C-grid finite volume scheme [47] with $\Delta x=1.0 \times 10^{4}, K=60, L=5.0 \times 10^{6}, D=5.0 \times 10^{3}, f=1.0 \times 10^{-4}$. The IMEX method described in $\S 3-\S 5$, SE method described in $\S$ Appendix A, as well as fourth order Runge-Kutta (RK4, used as a reference solution), and their shared components are implemented using GCC 4.7.3 [48], Open MPI 1.6.4 [49], Parallel netCDF 1.3.1 [50] and Trilinos 10.8.3 [51]. The computations for these two examples were performed in serial on an Apple $2.3 \mathrm{GHz}$ Core i7, $8 \mathrm{~GB} 1333 \mathrm{MHz}$ DDR3 with OS X 10.7.5. For the IMEX method, the preconditioner applica- 
Table 1: Timestep size, $\Delta t_{\mathrm{HO}}$, and relative nonlinear tolerance, $\tau_{r}$, for IMEX for problem $\S 6.1$.

\begin{tabular}{ccccccccccc}
$\Delta t_{\mathrm{LO}}$ & 1.875 & 3.75 & 7.5 & 15 & 30 & 60 & 120 & 240 & 480 & 960 \\
\hline$\tau_{r}$ & $10^{-6}$ & $10^{-6}$ & $10^{-5}$ & $10^{-5}$ & $10^{-4}$ & $10^{-3}$ & $10^{-3}$ & $10^{-2}$ & $10^{-2}$ & $10^{-1}$ \\
\hline
\end{tabular}

tion (42) is performed using algebraic multigrid via Trilinos ML [52]. For both examples we utilize the default settings for smoothed aggregation, in particular the operator is coarsened to 2 levels using a symmetric Gauss-Seidel smoother with 5 sweeps and no damping. At the coarsest level, there are 166 nonzeros in the matrix, and two applications of the Jacobi smoother with two V-cycle applications are employed. For the IMEX method, we choose an absolute nonlinear tolerance, $\tau_{a}=0$ and a minimum relative nonlinear tolerance, $\tau_{r}$, small enough for each timestep, $\Delta t_{\mathrm{LO}}$, to ensure second-order temporal accuracy. The values chosen for $\tau_{r}$ for each $\Delta t_{\mathrm{LO}}$ are given in Table 1. With this choice of tolerance, Newton's method converges in one iteration per timestep and one GMRES iteration per Newton iteration for both example problems. In addition, we use the parameters $\eta_{(0)}=.1, \eta_{\min }=1 \times 10^{-6}, \eta_{\max }=.01, \alpha=1.5$ and $\gamma=.9$ to control convergence of GMRES [43]. The SE method required 2 Picard iterations per timestep, $\Delta t_{\text {но }}$, to ensure second-order temporal accuracy, thus 2 Picard iterations were used throughout the study. Note that in the figures the timestep axis refers to the outer timestep: $\Delta t_{\mathrm{LO}}$ for IMEX and $\Delta t_{\mathrm{HO}}$ for SE.

\subsection{Problem 1: Surface perturbation, constant density, integration to 1 day}

The motivation for this problem is to compare CPU time, accuracy and efficiency of the IMEX and SE methods under purely barotropic motions. In this problem, there is no impact of the temperature and salinity on the gravity wave propagation.

Time scales in this problem are: $\Delta t_{g} \approx 45, \Delta t_{a} \approx 3 \times 10^{3}, \Delta t_{f} \approx 1 \times 10^{4}$ and $\Delta t_{d} \gg \Delta t_{f}$. This is the same problem configuration as problem 1 from [8]. To demonstrate convergence, we define numerical error for $U=\left[\begin{array}{lll}\mathbf{u} w & \eta & \varphi\end{array}\right]$ as $\left\|U-U_{\text {ref }}\right\|_{2}$, in terms of a reference solution, $U_{\text {ref }}$, computed with RK4 with $\Delta t=$ 1.875. The RK4 timestep was chosen such that a sufficiently high resolution reference solution could be used for verification of second-order convergence for the IMEX and SE methods.

Figure 1a shows error as a function of timestep size at $t=1$ day for IMEX and SE. Error is computed relative to the RK4 solution and clearly shows IMEX and SE to have second-order convergence. Figure 1a provides a verification of the expected order of accuracy of each method. It is important to note in this example that SE requires subcycling of the low order problem for $\Delta t_{\mathrm{LO}}>\Delta t_{g}$, while IMEX requires no subcycling of the high order problem as $\Delta t_{\mathrm{HO}}<\Delta t_{d}$ for all timesteps. Note that $\mathrm{SE}$ shows a transition zone between second-order slopes, which occurs at timesteps where subcycling is active. The transition reflects the fact that the error is dominated by the smaller (subcycling) timestep. 
Figure $1 \mathrm{~b}$ shows error as a function of serial CPU time for IMEX and SE, and shows SE to be twice as efficient as IMEX for $\Delta t_{\mathrm{Hо}}<\Delta t_{g}$. However, SE is up to six times more efficient as IMEX for a target accuracy for $\Delta t_{\mathrm{HO}}>\Delta t_{g}$, where the SE error is controlled by the smaller subcycling timesteps.

Figure 1c shows CPU time as a function of timestep size for both $\mathrm{HO}$ and LO components of IMEX and SE, and follows the cost analysis of (47) and (51). In particular, in both IMEX and SE methods, the dominant cost is in the HO solver. Both IMEX HO and SE HO scale well algorithmically over the range of timesteps. Since $\Delta t_{\mathrm{Hо}}$ is the same for both IMEX and SE, the number of calls to the HO solver is roughly the same for both IMEX and SE. The IMEX LO solver costs considerably less than the SE LO solver, and scales well algorithmically. By design of the IMEX algorithm, and since SE requires subcycling of the LO solver, IMEX makes fewer calls to the LO solver than SE. The algorithmic scaling observed in the implicit IMEX LO solver is made possible by effective preconditioning with $N_{\mathrm{G}} \approx N_{\mathrm{P}}$. The SE LO solver increases in cost with $\Delta t_{\mathrm{HO}}$ when $\Delta t_{\mathrm{HO}}>\Delta t_{g}$. This is due to the fact that, for SE, subcycling of the LO components is required when $\Delta t_{\mathrm{HO}}>\Delta t_{g}$, and $\Delta t_{\mathrm{LO}}$ is fixed at $\Delta t_{\mathrm{LO}}=\Delta t_{g}$, and more work is required for the LO time integration.

Figure 1d shows total CPU time (both HO and LO components) as a function of timestep size for IMEX and SE. It shows IMEX costs as much as 2.5 times more than SE over the range of timesteps, since the HO cost dominates. This is consistent with the heuristic (55) with $N_{\mathrm{FE}}=4\left(N_{\mathrm{G}}=N_{\mathrm{N}}=1\right), N_{\mathrm{P}}=2$ and $c_{s}=0 \mathrm{~m} / \mathrm{s}$ as this problem has no advection by design, which gives $C_{\mathrm{IMEX}} / C_{\mathrm{SE}} \approx$ 2 .

\subsection{Problem 2: Surface perturbation, linear temperature distribution with tem-} perature perturbation near surface, variable density, integration to 1 day

In this example, the initial condition for temperature is a linear distribution in depth with $\varphi_{1,1}=20.0$ and $\varphi_{1,60}=10.0$, combined with a perturbation in layers near the surface. The initial condition for salinity is a linear distribution in depth with $\varphi_{2,1}=30.0$ and $\varphi_{2,60}=26.0$. This initial condition is the same as problem 2 from [8] and is particularly motivated by [53]. With this initial condition, baroclinic motions develop immediately with $\Delta t_{d} \approx 300$. Thus, subcycling of the HO component of IMEX is required such that $\Delta t_{\mathrm{HO}}=\Delta t_{d}$ when $\Delta t_{\mathrm{LO}}>\Delta t_{d}$. We use the same solver tolerances and discretization as in $\S 6.1$.

Figure 2a shows error as a function of timestep size at $t=1$ day for IMEX and SE and clearly shows IMEX and SE to have second-order convergence. The SE method requires subcycling of the low order problem for $\Delta t_{\mathrm{LO}}>\Delta t_{g}$, while IMEX requires subcycling of the high order problem only for $\Delta t_{\mathrm{Hо}}>\Delta t_{d}$. Figure 2b shows error as a function of CPU time for IMEX and SE.

Figure 2c shows CPU time as a function of timestep size for both $\mathrm{HO}$ and LO components of IMEX and SE, and follows the cost analysis of (47) and (51). Figure $2 \mathrm{c}$ shows the IMEX HO component to scale ideally with a slight increase in cost for $\Delta t_{\mathrm{LO}}>\Delta t_{d}$, since $\Delta t_{\mathrm{HO}}=\Delta t_{d}$ is fixed and the HO solver requires more work. However, even though the IMEX HO component is being subcycled, 
there is only a modest increase in CPU cost. The IMEX LO component scales independently of $\Delta t_{d}$ due to effective preconditioning. The SE HO component scales well; the SE LO component scales well for $\Delta t_{\mathrm{HO}}<\Delta t_{g}$, but the cost increases for $\Delta t_{\mathrm{HO}}>\Delta t_{g}$ since $\Delta t_{\mathrm{LO}}=\Delta t_{g}$. As in $\S 6.1$, the CPU cost of both the IMEX and SE methods are dominated by the HO components. Figure 2d shows total CPU time (both $\mathrm{HO}$ and $\mathrm{LO}$ components) as a function of timestep size for IMEX and SE and shows both methods to scale well, although IMEX to costs more than SE over the range of timesteps. Using the heuristic (55) with $N_{\mathrm{FE}}=4, N_{\mathrm{P}}=2, K=60, c_{f} \approx 225 \mathrm{~m} / \mathrm{s}$ and $c_{s} \approx 33 \mathrm{~m} / \mathrm{s}$, we find $C_{\mathrm{IMEX}} / C_{\mathrm{SE}} \approx 2$. Indeed, we see that computational costs of IMEX are 2.5 times that of SE over the range of timesteps.

Our results show the IMEX algorithm is algorithmically scalable and secondorder accurate, with serial computational costs about 2.5 times that of the SE method. In $\S 7$, we present a communication staging strategy that favors particularly the IMEX approach and can significantly reduce the cost of the IMEX HO solver in parallel.

\section{Communication staging}

Our implementation of both IMEX and SE utilizes domain decomposition as the basis of parallelization, with each processor computing on a single subdomain. Each subdomain has halo cells which overlap its neighbor subdomain cells. These halo cells contain the information necessary for each subdomain to compute derivatives. Traditional single-halo implementations of time-dependent simulations perform a single halo exchange or communication once per timestep for single stage methods and once per stage in multi-stage methods. Most ocean models, including ours, utilize domain decomposition in the horizontal surface only, with each subdomain extruded vertically in depth. Thus there is horizontal halo exchange required on each layer. Our communication staging strategy aggregates the network communications of halo cells necessary for multiple explicit updates by increasing the width of the halo band relative to the number of staged timesteps, similar to [34]. In our implementation, the halo width is increased and the necessary halo communication for multiple timesteps is performed in advance at the beginning of the timestep, followed by computation of multiple, possibly subcycled, timesteps. This strategy particularly favors our IMEX method over SE, due to the fact that IMEX subcycles the HO components over longer timesteps, rather than subcycling the LO components over smaller timesteps.

In $\S 7.1$, we examine the communication staging mechanisms and develop a model for a simple case. We extend the strategy to IMEX and SE for ocean simulation in $\S 7.2$, and compare communication staging to single-halo implementations in $\S 8$.

\subsection{Communication staging model}

The goal of communication staging is to reduce the effects of interconnect latency between nodes that is inherent in spatially discretized, time-dependent 


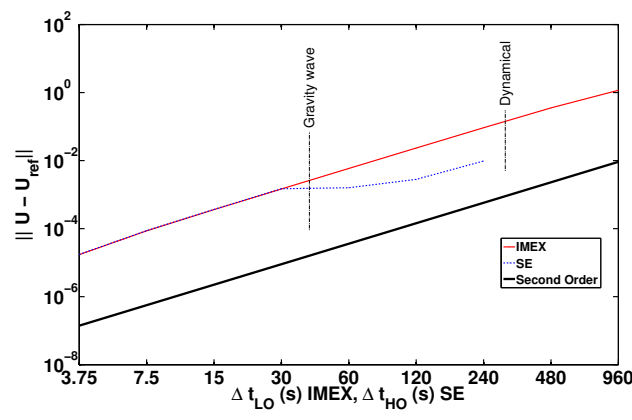

(a)

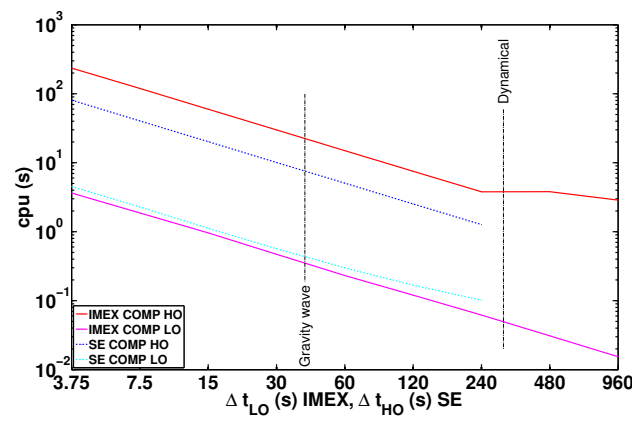

(c)

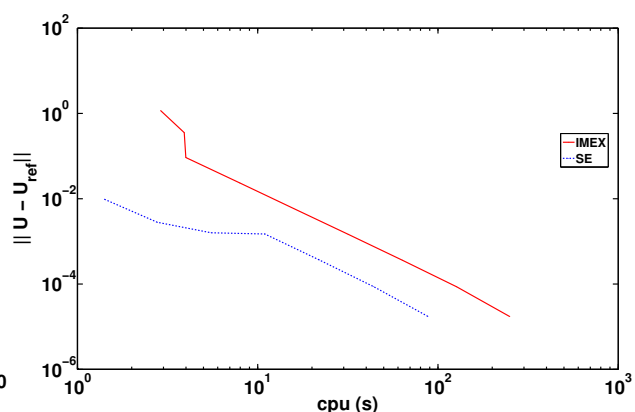

(b)

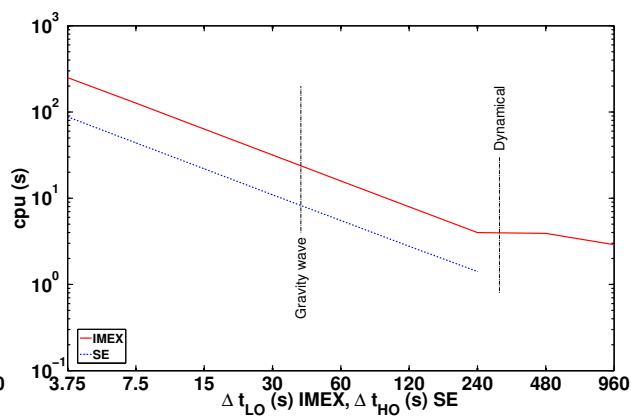

(d)

Figure 2: Convergence and timing results for problem $\S 6.2$. (a) Timestep size vs. error for IMEX and SE. Error rate is computed relative to a reference solution $U_{\text {ref }}$ obtained by fourthorder RK4 with $\Delta t=1.875$. (b) CPU vs. error for IMEX and SE. (c) Timestep size vs. CPU for HO and LO components of IMEX and SE. (d) Timestep size vs. total CPU for IMEX and SE.

codes; similar in intent to [31, 32] and [33]. Each time we perform a network communication, we pay some overhead for starting and ending the communication, and then a penalty for communicating the data itself. By extending the width of the halo (by a variable amount based on algorithmic needs, as opposed to a fixed amount as in [30]), we pay only one overhead penalty per outer timestep. While we consider a network bus in our examples, our analysis extends to any high-latency bus.

We observe that a few structural changes are necessary to convert single-halo code to an effective staged-communication code. First, we need a mutable runtime parameter to express the width of the halo region. Second, for efficiency, we need to perform extra book-keeping in our computational routines so that they compute over a width relative to the current position within a block of staged iterations.

Consider a horizontal 1D vector of local length $l$ with halo width $h$ staged for $n$ timesteps, and assume a simple derivative stencil with a halo overlap of 
width unity for each timestep. Hence $h=n$. We can perform operations (e.g. multiplies, adds) over the entire halo at each timestep, for a cost (in operations) of $l+h^{2}$ to process the entire vector, or we can perform a minimum amount of operations, reducing the effective halo width each timestep, for a cost of $l+\frac{h}{2}(h+1)$. A reason we may choose the former approach of performing extra operations is staging on an accelerator, where one might consider branching to be a larger penalty than consuming additional flops. However, in this work, we choose the latter approach and always perform the bookkeeping necessary to ensure we compute only over the portion of the vector necessary for each timestep.

Extending the model to a horizontal 2D vector, with square domain of local width $l$, and $h=n$, the amount of operations to process the entire vector is

$$
h(l+2 h)^{2}=h l^{2}+4 h^{2} l+4 h^{3},
$$

and the amount of minimal operations would be

$$
\begin{aligned}
\sum_{n=1}^{h}(l+2 n)^{2} & =\frac{2}{3} h+2 h^{2}+\frac{4}{3} h^{3}+2 h l+2 h^{2} l+h l^{2}, \\
& \approx h l^{2}+2 h^{2} l+\frac{4}{3} h^{3} .
\end{aligned}
$$

In practice, $l \gg h$, thus use of the latter method potentially reduces the computational intensity to process the halo elements by one half. Similarly, for 3D, we have

$$
h(l+2 h)^{3}=8 h^{4}+12 h^{3} l+6 h^{2} l^{2}+h l^{3},
$$

operations to process the entire vector, and

$$
\begin{aligned}
\sum_{n=1}^{h}(l+2 n)^{3} & =2 h^{2}+4 h^{3}+2 h^{4}+2 h l+6 h^{2} l+4 h^{3} l+3 h l^{2}+3 h^{2} l^{2}+h l^{3} \\
& \approx 2 h^{4}+4 h^{3} l+3 h^{2} l^{2}+h l^{3}
\end{aligned}
$$

minimal operations to process the entire vector. In general, communication staging potentially reduces the computational intensity to process the halo elements by one half.

\subsection{Communication staging IMEX and SE}

We discuss the implementation details of communication staging for the SE method as presented in $\S$ Appendix A, and the IMEX method detailed in $\S 3$. We also provide models for communication cost per timestep for single-halo and staged communication for both methods. We model communication cost in terms of $\mathrm{HO}$ and $\mathrm{LO}$ communication. $\mathrm{HO}$ communication must communicate data for $\mathbf{u}_{k}, w_{k}, \rho_{k}$ and $\varphi_{i, k}, i=1,2$ in all $k=1, \ldots, K$ layers of the ocean model, while an LO communication must communicate data for $\eta$ and $\overline{\mathbf{u}}$ in the 


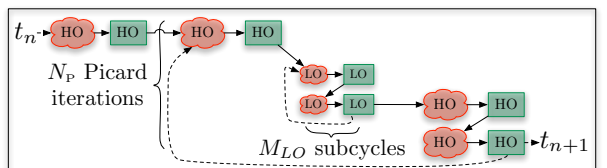

Split Explicit - Single Halo

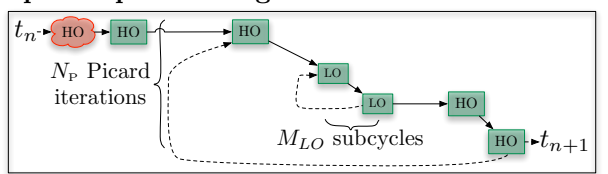

Split Explicit - Staged

HOS 103 Communication

(a)

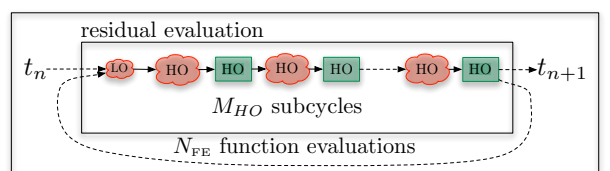

IMEX - Single Halo

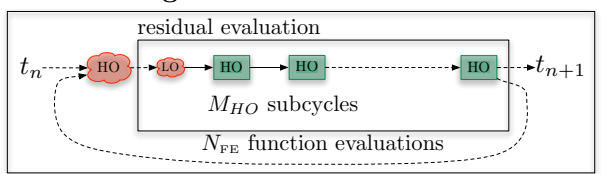

IMEX - Staged

(HO) 100 Communication

(b)

Table 2: Communication counts per timestep, $c$, and halo width, $h$, for LO and HO in SE, single-halo and staged-communication as a function of Picard iterations, $N_{\mathrm{P}}$, and subcycles, $M_{\mathrm{LO}}$.

\begin{tabular}{ccccc} 
& \multicolumn{2}{c}{ Single-halo } & \multicolumn{2}{c}{ Staged } \\
& HO & LO & HO & LO \\
\hline \# comm/timestep, $c$ & $3 N_{\mathrm{P}}+1$ & $4 N_{\mathrm{P}} M_{\mathrm{LO}}$ & 1 & 1 \\
\hline halo width, $h$ & 1 & 1 & $N_{\mathrm{P}}\left(4 M_{\mathrm{LO}}+3\right)+1$ & $N_{\mathrm{P}}\left(4 M_{\mathrm{LO}}+3\right)+1$ \\
\hline
\end{tabular}

surface, $k=1$, only. Thus HO communication is much more costly than LO communication.

Figure 3 shows traditional single-halo and staged communication workflows for SE and IMEX. Single calls to communication and computation for $\mathrm{HO}$ and LO components are depicted by clouds and rectangles respectively. Note that a cloud represents a single call to communication only, and does not specify the amount of data, or halo width, communicated. Recall that a single HO timestep of SE consists of an $\mathrm{HO}$ function evaluation; subcycling the LO system $M_{\mathrm{LO}}$ steps, via a predictor corrector method, followed by a predictor corrector integration of the $\mathrm{HO}$ system, enclosed in $N_{\mathrm{P}}$ Picard iterations. In the traditional, single-halo implementation, we have an $\mathrm{HO}(\mathrm{LO})$ communication before every HO (LO) computation. Thus, single-halo SE performs $c=4 N_{\mathrm{P}} M_{\mathrm{LO}} \mathrm{LO}$ communications per timestep and $c=3 N_{\mathrm{P}}+1 \mathrm{HO}$ communications per timestep, each with a halo width of $h=1$.

In contrast, staged SE performs only one $\mathrm{HO}$ and LO communication step (Figure 3a shows only the $\mathrm{HO}$ communication as it dominates), with a halo width of $h=N_{\mathrm{P}}\left(4 M_{\mathrm{LO}}+3\right)+1$ per timestep. This halo width is derived from the HO initial communication, 1, the HO communication at the start of each Picard step, $N_{\mathrm{P}}$, the LO communications for the subcyling, $4 N_{\mathrm{P}} M_{\mathrm{LO}}$, and the 
Table 3: Communication counts per timestep, $c$, and halo width, $h$, for LO and $\mathrm{HO}$ in IMEX, single-halo and staged-communication as a function of Newton iterations, $N_{\mathrm{N}}$, function evaluations, $N_{\mathrm{FE}}$, and subcycles, $M_{\mathrm{HO}}$.

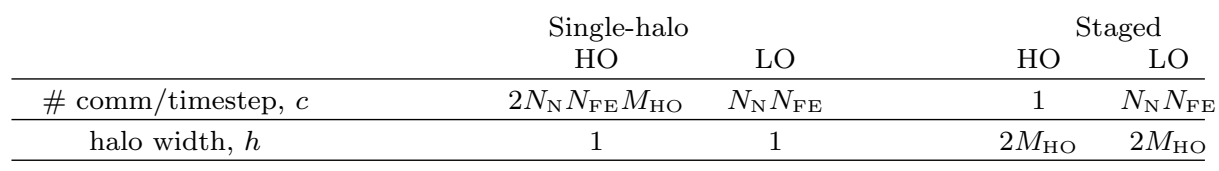

$\mathrm{HO}$ communication for the $\mathrm{HO}$ predictor-corrector stage, $2 N_{\mathrm{P}}$. The single-halo and staged-communication SE workflows are shown schematically in Figure 3a. Table 2 summarizes the number of HO and LO communications per timestep, $c$, the amount of communication required in terms of halo width, $h$, for single-halo and staged-communication SE.

A single LO timestep of the IMEX method requires $N_{\mathrm{N}}$ Newton iterations, each of which requires $N_{\mathrm{FE}} \mathrm{HO}$ residual evaluations. We note that in the remainder of the manuscript we use $N_{\mathrm{FE}}$ to denote the number of HO function evaluations per Newton iteration. Each residual evaluation performs $M_{\text {Hо }}$ predictor-corrector subcycles of the HO problem. For the single-halo IMEX implementation, outside of the communications of the nonlinear solver package, there is one LO communication per residual evaluation, and one HO communication for each HO subcycle, with all communications of halo width $h=1$. Therefore for single-halo, we have $c=2 N_{\mathrm{N}} N_{\mathrm{FE}} M_{\mathrm{HO}}$ for $\mathrm{HO}$ and $c=N_{\mathrm{N}} N_{\mathrm{FE}}$ for LO.

For staged IMEX, the largest benefit is to perform all of the HO communication once per timestep, rather than once per subcycle. However, LO communication must still be performed once per residual evaluation. Therefore, we have $c=1$ for $\mathrm{HO}$ communication and $c=N_{\mathrm{N}} N_{\mathrm{FE}}$ for LO communication. A halo width $h=2 M_{\mathrm{HO}}$ is required for both $\mathrm{HO}$ and $\mathrm{LO}$ communication.

In fact, for staged IMEX with $M_{\mathrm{HO}}=1$ (no HO subcycling), we still see a benefit in communication costs over SE in the regime where LO subcycling is required. In this case the required HO communication for IMEX is $h=2$, and for $\mathrm{SE}$ the required $\mathrm{HO}$ communication is $h=N_{\mathrm{P}}\left(4 M_{\mathrm{LO}}+3\right)+1$. The singlehalo and staged communication IMEX are shown schematically in Figure 3b. Table 3 summarizes the number of $\mathrm{HO}$ and LO communications per timestep, $c$, the amount of communication required in terms of halo width, $h$, for single-halo and staged communication for IMEX.

There is an obvious compromise in communication staging. Communication is performed less often, however more data needs to be communicated. From Table 3, we see that communication staging will benefit IMEX as an absolute reduction in $\mathrm{HO}$ communication calls by a factor of $2 N_{\mathrm{N}} N_{\mathrm{FE}} M_{\mathrm{HO}}$. In our studies, we have $N_{\mathrm{N}}=1, N_{\mathrm{FE}}=4$ and $M_{\mathrm{HO}} \ll M_{\mathrm{LO}}$. However, halo width required for each communication is increased by $2 M_{\mathrm{HO}}$. For SE, we see that staging appears to be a burden in that the halo width of the HO communication is dominated by the number of LO subcycles. Since HO communication is more costly than 
LO communications and in our studies we typically have $N_{\mathrm{P}}=N_{\mathrm{N}}=1$ and $M_{\mathrm{HO}} \ll M_{\mathrm{LO}}$, we expect communication staging to perform more effectively in IMEX than SE.

\section{Communication staging results}

We present two example problems to demonstrate the effects of communication staging in IMEX and SE. We perform a weak scaling study to evaluate effectiveness of our communication staging strategy for both IMEX and SE. The computations were performed on the Mustang system at Los Alamos National Laboratory which is composed of 1600 nodes; each node is comprised of two 12-Core AMD Opteron model 6176 processors at $2.3 \mathrm{GHz}$, and $64 \mathrm{~GB}$ of DDR3 memory, with software: TOSS 1.4, GCC 4.7.0, Trilinos 10.8.5 and OpenMPI 1.6.5. The problem configuration is that of $\S 6.2$. In order to demonstrate network communication effects, the examples in this section were run using one single-threaded MPI rank per node.

\subsection{Problem 3: Effects of communication staging SE and IMEX}

In the first example, we study the reduction in communication costs associated with raw data movement for staged SE and IMEX. We compare the single-halo implementation and the staged-communication strategy for SE and IMEX on $\{1,2,3,4,6,8,12,16,24,48,96,192,384,768\}$ nodes in two weak scaling configurations. The first configuration, denoted $5 \%$, fixes the ratio $h: l$ to $1: 9$, and the second configuration, denoted $1 \%$, fixes the ratio to $1: 49$. The width of a processing element's subdomain is determined by the required halo width of one staged outer timestep (Tables 2-3). The required halo width of one staged outer timestep is multiplied by 20 for the $5 \%$ configuration or 100 for the $1 \%$ configuration. Thus, SE and IMEX will not have the same domain width. However, the cost of communication staging is evaluated for each method in a comparable fashion. The motivation behind the two configurations is to demonstrate that reducing the surface area of the extended halo relative to the portion

of the distributed vector owned by a partition reduces the computational cost of performing the additional work from the staged halo. Our expectation is that, as the ratio $h: l$ decreases, the relative communication efficiency will increase. The communication staging strategy has two effects: the ability to reduce network communication frequency, and the ability to increase the computational intensity in between network communications.

Figure 4a shows relative percent halo update plus communication as a function of number of nodes for the SE method. We define halo update plus communication time as the sum of MPI communication time and time required for halo update. Relative percent halo update plus communication is defined as the ratio of halo update plus communication to the sum of update plus communication time and compute time. We believe that this approach serves as a good proxy for measuring relative communication cost without the need to instrument Trilinos. Using this measurement, we expect that the cost is dominated by data fill 


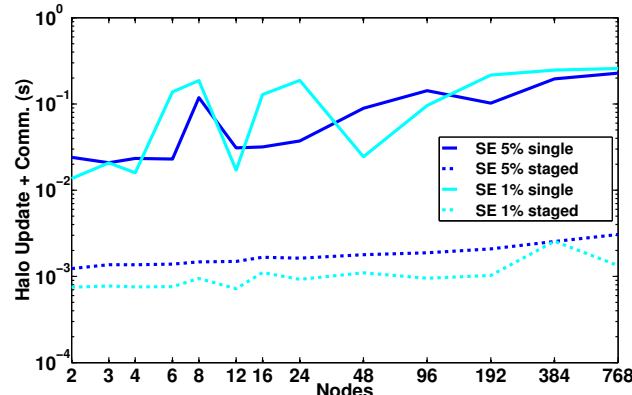

(a)

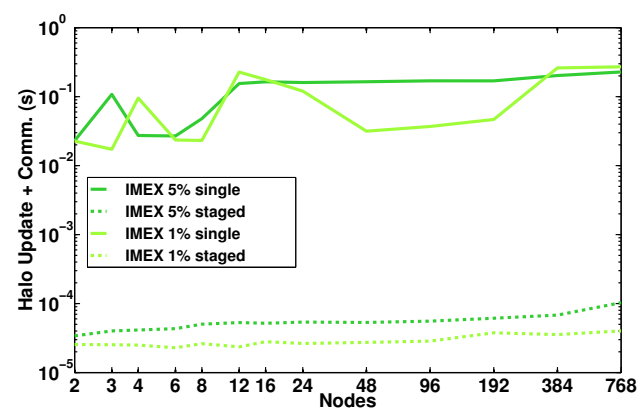

(c)

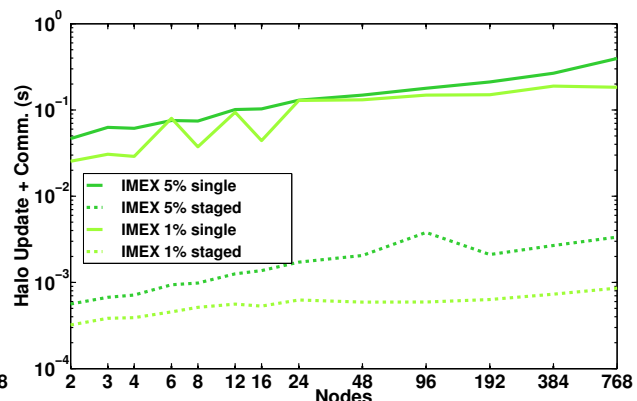

(b)

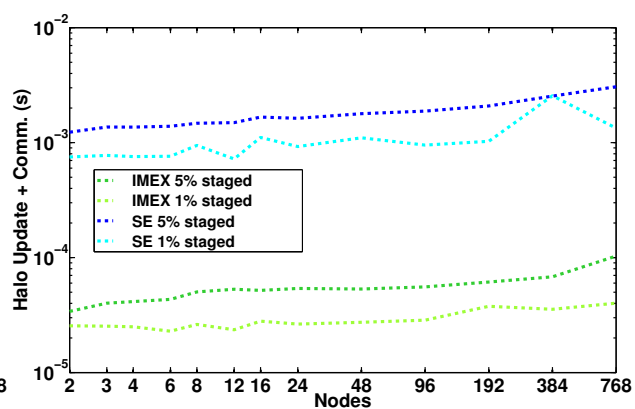

(d)

Figure 4: Relative percent halo update plus communication, traditional single-halo vs. stagedcommunication. (a) SE with $\Delta t_{\mathrm{HO}}=60\left(\Delta t_{\mathrm{LO}}=\Delta t_{g}\right), 1-768$ nodes. (b) IMEX with $\Delta t_{\mathrm{LO}}=60\left(\Delta t_{\mathrm{HO}}=\Delta t_{\mathrm{LO}}\right) 1$ subcycle, $1-768$ nodes. (c) IMEX with $\Delta t_{\mathrm{LO}}=480\left(\Delta t_{\mathrm{HO}}=\right.$ 60) 8 subcycles, $1-768$ nodes. (d) IMEX with $\Delta t_{\mathrm{LO}}=480\left(\Delta t_{\mathrm{HO}}=60\right) 8$ subcycles, and SE with $\Delta t_{\mathrm{HO}}=60\left(\Delta t_{\mathrm{LO}}=\Delta t_{g}\right), 1-768$ nodes.

at low node counts, and that the cost is dominated by communication at higher node counts. Figure $4 \mathrm{a}$ shows that communication staging provides between one and two orders of magnitude reduction in relative percent halo update plus communications as compared to the traditional single-halo implementation for the SE method.

The same study was repeated with IMEX with $\{1,8\}$ subcycles. Figure $4 \mathrm{~b}$ shows that communication staging produces approximately two orders of magnitude reduction in relative percent halo update plus communications versus the traditional single-halo implementation.

Figure $4 \mathrm{c}$ shows that increasing the number of subcycles to 8 yields between one and two additional orders-of-magnitude reduction in relative percent halo update plus communication.

Figure 4d shows a direct comparison of IMEX with 8 subcycles and SE on 1-768 nodes. We note that, while staging is effective in reducing the relative percent halo update plus communication for both IMEX and SE, it is most 


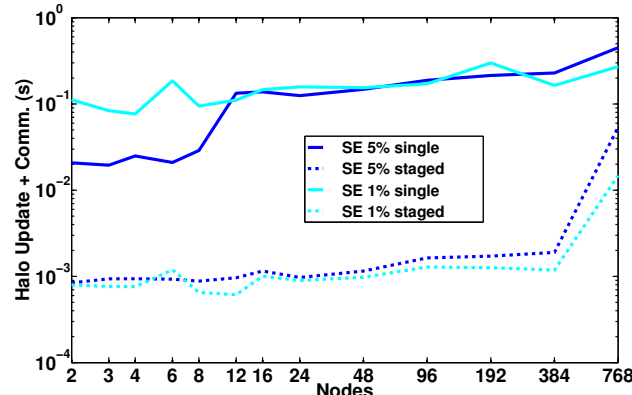

(a)

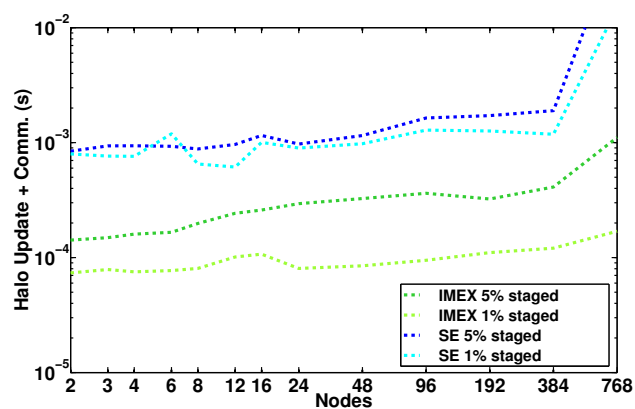

(c)

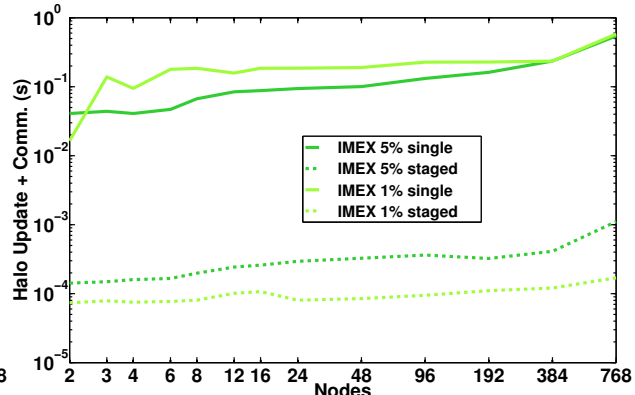

(b)

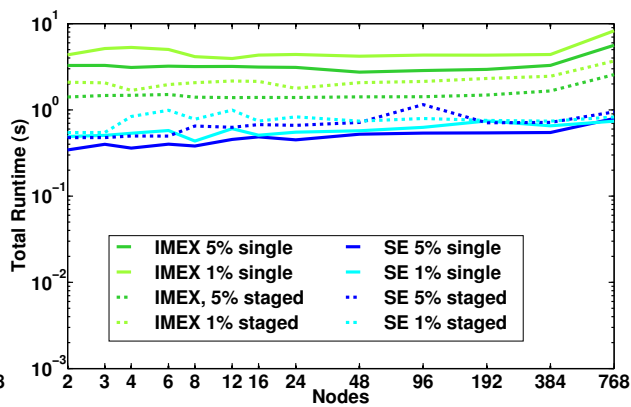

(d)

Figure 5: Relative percent halo update plus communication, traditional single-halo vs. stagedcommunication for problem $\$ 8.2$. (a) SE with $\Delta t_{\mathrm{HO}}=900\left(\Delta t_{\mathrm{LO}}=\Delta t_{g}\right), 1-768$ nodes. (b) IMEX with $\Delta t_{\mathrm{LO}}=900\left(\Delta t_{\mathrm{HO}}=\Delta t_{d}\right)$, 1-768 nodes. (c) IMEX with $\Delta t_{\mathrm{LO}}=900$ $\left(\Delta t_{\mathrm{HO}}=\Delta t_{d}\right)$, and SE with $\Delta t_{\mathrm{HO}}=960\left(\Delta t_{\mathrm{LO}}=\Delta t_{g}\right), 1-768$ nodes. (d) Total runtime per degree of freedom for IMEX with $\Delta t_{\mathrm{LO}}=900\left(\Delta t_{\mathrm{HO}}=\Delta t_{d}\right)$, and SE with $\Delta t_{\mathrm{HO}}=900$ $\left(\Delta t_{\mathrm{LO}}=\Delta t_{g}\right), 1-768$ nodes.

effective for IMEX, and amplified by increasing the number of subcycles. We observe over an order of magnitude savings in relative percent halo update plus communication in IMEX over SE.

8.2. Problem 4: Surface perturbation, linear temperature distribution with temperature perturbation near surface, variable density, integration with large $\Delta t$

In this example, we repeat the problem of $\S 6.2$ with $\Delta t_{\mathrm{HO}}=900\left(\Delta t_{\mathrm{LO}}=\right.$ $\left.\Delta t_{g}\right)$ for SE and $\Delta t_{\mathrm{LO}}=900\left(\Delta t_{\mathrm{HO}}=\Delta t_{d}\right)$ for IMEX. With this choice of outer timestep size, the SE LO component must be subcycled $M_{\mathrm{LO}}=30$ times and the IMEX HO component must be subcycled $M_{\mathrm{HO}}=3$ times. The motivation for this problem is to compare the relative communication cost for IMEX and SE for integration at timesteps larger than $\Delta t_{g}$ and $\Delta t_{d}$, i.e. applicable to thermohaline-circulation-class problems. 
Figure 5a shows SE with $\Delta t_{\mathrm{HO}}=900\left(\Delta t_{\mathrm{LO}}=\Delta t_{g}\right), 1-768$ nodes, singlehalo and staged communication. The figure shows that, for the single-halo implementation, the relative percent halo update plus communication increases with number of nodes. In particular, the relative percent halo update plus communication is $20 \%-40 \%$ for single-halo implementation for 768 nodes. When staged communication is utilized for SE, the relative percent halo update plus communication is reduced to $.1 \%-.4 \%$ for 768 nodes.

Figure 5b shows IMEX with $\Delta t_{\mathrm{LO}}=900\left(\Delta t_{\mathrm{HO}}=\Delta t_{d}\right), 1-768$ nodes, single-halo and staged communication. The figure shows that for the singlehalo implementation, the relative percent halo update plus communication is approximately $40 \%$ for 768 nodes. However, the relative percent halo update plus communication is reduced to $.001 \%^{-} .01 \%$ for 768 nodes when staged communication is utilized. Figure $5 \mathrm{c}$ shows that the communication staging strategy reduces the relative communication costs for IMEX by an order of magnitude, or more, over that of SE.

Figure 5d shows the total computation time per degree of freedom for SE with $\Delta t_{\mathrm{HO}}=900\left(\Delta t_{\mathrm{LO}}=\Delta t_{g}\right)$ and IMEX with $\Delta t_{\mathrm{LO}}=900\left(\Delta t_{\mathrm{HO}}=\Delta t_{d}\right)$, 1-768 nodes, single-halo and staged communication. The figure confirms the weak scaling nature of the test, with computation rapidly becoming a fixed cost per method, choice of staged versus single-halo, and type of halo size. Figure 5d also shows that staging increased the computational time for SE, while reducing that of IMEX.

Our results show that, with an effective communication staging strategy and subcycling of the HO problem, our IMEX method in a parallel environment can decrease the relative communication by more than two orders of magnitude over the split-explicit approach. This makes IMEX ideally suited for heterogeneous massively-parallel environments, where communication costs continue to grow and are dominant relative to floating-point costs. The increase in savings of relative communication costs of the IMEX method over SE in the regime of large timesteps and large number of nodes is made possible by the ability to subcycle the HO component within an implicit treatment of the LO component. While our results have been obtained in $2 \mathrm{D}$, the analysis in $\S 7.1$ suggests similar savings in relative communication costs in $3 \mathrm{D}$.

\section{Conclusions}

This study has demonstrated a communication-avoiding IMEX method for ocean free-surface z-level ocean simulation. The formulation treats the barotropic (LO) system implicitly with a preconditioned JFNK method. The implicit formulation allows algorithmically scalable time integration with timesteps much larger than the traditional explicit limit. The algorithmic scalability of the method is enabled by effective preconditioning of the barotropic system. The baroclinic (HO) system is subcycled explicitly and nonlinearly eliminated within the JFNK residual evaluation. In addition, a communication staging strategy is developed for parallel environments that not only improves the performance of the split-explicit method, but particularly favors the IMEX method. The 
explicit treatment of the baroclinic system enables an effective communication staging strategy that allows $\mathrm{HO}$ communication to be performed once per LO timestep, thus reducing communication costs for the IMEX method over traditional split-explicit methods. Our method differs markedly from traditional split-explicit approaches, where the baroclinic HO system is advanced with a long timestep, $\Delta t_{\mathrm{HO}}$, and the barotropic LO system is subcycled with short timesteps, $\Delta t_{\mathrm{LO}}$, restricted by a barotropic CFL limit, within a single $\mathrm{HO}$ timestep.

Our results show the IMEX algorithm is algorithmically scalable and secondorder accurate. Although the serial cost of IMEX is 2.5 more than SE in our test problems, we have shown that in parallel with an effective communication staging strategy and subcycling of the HO problem, the IMEX approach results in more than one order of magnitude decrease in relative communication cost versus the split-explicit approach. Thus, IMEX is ideally suited for exascale environments, where communication costs continue to grow and are dominant relative to floating point costs.

\section{Acknowledgements}

This work was sponsored by the Los Alamos National Laboratory Directed Research and Development Program and by the US Department of Energy Office of Science. This work was performed under US government contract DEAC52-06NA25396 for Los Alamos National Laboratory, which is operated by Los Alamos National Security, LLC, for the US Department of Energy (LAUR-14-26791).

\section{Appendix A. Split-explicit method}

The implementation of SE used in this manuscript is similar to the implementations of [37] and [36] with theoretical development given in $[4,5]$. The method is shown schematically in Fig. 3a. The SE method differs from IMEX as the HO solver is the outside solver with the LO components subcycled within the HO solver $\left(\Delta t_{\mathrm{LO}}<\Delta t_{\mathrm{HO}}\right)$. Recall that in the IMEX method the LO solver is the outside solver with HO components subcycled within the LO solver, $\left(\Delta t_{\mathrm{LO}}>\Delta t_{\mathrm{HO}}\right)$. In this approach the $\mathrm{HO}$ problem is given by (1)-(3), (5)-(9) and the LO problem given by (15) and

$$
\frac{\partial \overline{\mathbf{u}}}{\partial t}+G_{1}\left(\varphi_{i, k}, \eta\right)+\overline{F\left(\mathbf{u}_{k}, \eta, \varphi_{i, k}\right)}=0, \quad k=1, \ldots, K,
$$

where

$$
F\left(\mathbf{u}_{k}, \eta, \varphi_{i, k}\right)=L_{1}\left(\mathbf{u}_{k}, \eta\right)+f \mathbf{u}_{k}{ }^{\perp}+G_{2}\left(\varphi_{i, k}\right), \quad k=1, \ldots, K,
$$

and (A.1) is obtained via application of $(14)$ to $(1)-(3)$, see $[4,5]$. In the remainder of the text we omit the use of subscripts for vertical level and tracer 
indexing when the meaning is clear. As in $\S 3$, we utilize subscripts for $\mathrm{HO}$ timestep index and superscripts for subcycled LO timestep index.

In general, an $\mathrm{HO}$ timestep from time $t_{n}$ consists of subcycling the LO system $M_{\mathrm{LO}}$ steps, followed by integration of the HO system to time $t_{n+1}$. To ensure convergence, these two steps are enclosed in a Picard iteration [38]. In particular, the LO system is discretized via a predictor-corrector method with initial values $\mathbf{u}_{(0)}=\mathbf{u}_{n}, \eta_{(0)}=\eta_{n}, \varphi_{(0)}=\varphi_{n}, \overline{\mathbf{u}}^{0}=\overline{\mathbf{u}}_{n}$, and $\eta^{0}=\eta_{n}$, and subcycled $m=0, \ldots, M_{\mathrm{LO}}-1$ steps, where $\Delta t_{\mathrm{HO}}=M_{\mathrm{LO}} \Delta t_{\mathrm{LO}}$ and $\Delta t_{\mathrm{LO}} \approx \Delta t_{g}$, as follows:

$$
\begin{gathered}
\overline{\mathbf{u}}^{*}-\overline{\mathbf{u}}^{m}+\Delta t_{\mathrm{LO}} G_{1}\left(\varphi_{n}, \eta^{m}\right) \\
+\frac{\Delta t_{\mathrm{LO}}}{2}\left(\overline{F\left(\mathbf{u}_{n}, \eta_{n}, \varphi_{n}\right)}+\overline{F\left(\mathbf{u}_{(i)}, \eta_{(i)}, \varphi_{(i)}\right)}\right)=0, \\
\eta^{*}-\eta^{m}+\frac{\Delta t_{\mathrm{LO}}}{2}\left(\nabla \cdot\left(\overline{\mathbf{u}}^{m} H\left(\eta^{m}\right)\right)+\nabla \cdot\left(\overline{\mathbf{u}}^{*} H\left(\eta^{m}\right)\right)\right)=0, \\
\overline{\mathbf{u}}^{m+1}-\overline{\mathbf{u}}^{m}+\frac{\Delta t_{\mathrm{LO}}}{2}\left(G_{1}\left(\varphi_{n}, \eta^{m}\right)\right. \\
\left.+G_{1}\left(\varphi_{n}, \eta^{*}\right)+\overline{F\left(\mathbf{u}_{n}, \eta_{n}, \varphi_{n}\right)}+\overline{F\left(\mathbf{u}_{(i)}, \eta_{(i)}, \varphi_{(i)}\right)}\right)=0, \\
\eta^{m+1}-\eta^{m}+\frac{\Delta t_{\mathrm{LO}}}{2}\left(\nabla \cdot\left(\overline{\mathbf{u}}^{m} H\left(\eta^{m}\right)\right)+\nabla \cdot\left(\overline{\mathbf{u}}^{m+1} H\left(\eta^{*}\right)\right)\right)=0,
\end{gathered}
$$

where the subscript $(i)$ denotes the $i$ th Picard iterate, $\eta^{M_{\mathrm{LO}}}=\eta_{(i+1)}$ and $\overline{\mathbf{u}}^{M_{\mathrm{LO}}}=\overline{\mathbf{u}}_{(i+1)}$. The HO problem is integrated with a predictor-corrector method as follows:

$$
\begin{gathered}
\mathbf{u}_{*}-\mathbf{u}_{n}+\Delta t_{\mathrm{HO}} F\left(\mathbf{u}_{n}, \eta_{n}, \varphi_{n}\right)+\frac{\Delta t_{\mathrm{HO}}}{2}\left(G_{1}\left(\varphi_{n}, \eta_{n}\right)+G_{1}\left(\varphi_{n}, \eta_{(i+1)}\right)\right)=0, \\
h\left(\eta_{(i+1)}\right) \varphi_{*}-h_{n} \varphi_{n}+\frac{\Delta t_{\mathrm{HO}}}{2}\left(L_{2}\left(\varphi_{n}, \mathbf{u}_{n}, \eta_{n}\right)+L_{2}\left(\varphi_{(i)}, \mathbf{u}_{(i)}, \eta_{(i)}\right)\right)=0, \\
\mathbf{u}_{\dagger}-\mathbf{u}_{n}+\frac{\Delta t_{\mathrm{HO}}}{2}\left(F\left(\mathbf{u}_{n}, \eta_{n}, \varphi_{n}\right)+F\left(\mathbf{u}_{*}, \eta_{(i+1)}, \varphi_{*}\right)\right. \\
\left.+G_{1}\left(\varphi_{n}, \eta_{n}\right)+G_{1}\left(\varphi_{n}, \eta_{(i+1)}\right)\right)=0, \\
h\left(\eta_{(i+1)}\right) \varphi_{(i+1)}-h_{n} \varphi_{n}+\frac{\Delta t_{\mathrm{HO}}}{2}\left(L_{2}\left(\varphi_{n}, \mathbf{u}_{n}, \eta_{n}\right)+L_{2}\left(\varphi_{*}, \mathbf{u}_{\dagger}, \eta_{(i+1)}\right)\right)=0
\end{gathered}
$$

and

$$
\mathbf{u}_{(i+1)}-\mathbf{u}_{\dagger}-\overline{\mathbf{u}}_{\dagger}+\overline{\mathbf{u}}_{(i+1)}=0
$$

where (A.11) ensures consistency with the LO problem. The Picard iteration is terminated at iteration $i=I$ upon convergence in some norm or with a finite 
number of given iterations and we have a completed timestep with $\mathbf{u}_{n+1}=$ $\mathbf{u}_{(i+1)}, \varphi_{n+1}=\varphi_{(i+1)}$, and $\eta_{n+1}=\eta_{(i+1)}$. In our studies, we select $I$ such that second order temporal convergence is attained. Picard iteration is often necessary for greater than first order temporal convergence when coupling $\mathrm{HO}$ and LO problems explicitly [54]. In practice, the ocean modelling community often chooses $I=2[36]$.

\section{References}

[1] M. Maltrud, J. McClean, An eddy resolving global 1/10 ocean simulation, Ocean Model. 8 (2005) 31-54.

[2] J. McClean, D. Bader, F. Bryan, M. Maltrud, J. Dennis, A. Mirin, P. Jones, Y. Kim, D. Ivanova, M. Vertenstein, et al., A prototype two-decade fullycoupled fine-resolution CCSM simulation, Ocean Model. (2011).

[3] J. Dukowicz, R. Smith, Implicit free-surface method for the Bryan-CoxSemtner ocean model, J. Geophys. Res. 99 (1994) 7991-8014.

[4] R. Hallberg, Stable split time stepping schemes for large-scale ocean modeling, J. Comput. Phys. 135 (1997) 54-65.

[5] R. Higdon, R. de Szoeke, Barotropic-baroclinic time splitting for ocean circulation modeling, J. Comput. Phys. 135 (1997) 30-53.

[6] E. Bernsen, H. Dijkstra, J. Thies, F. Wubs, The application of Jacobianfree Newton-Krylov methods to reduce the spin-up time of ocean general circulation models, J. Comput. Phys. 229 (2010) 8167-8179.

[7] H. Dijkstra, H. Oksuzoglu, F. Wubs, E. Botta, A fully implicit model of the three-dimensional thermohaline ocean circulation, J. Comput. Phys. 173 (2001) 685-715.

[8] C. Newman, D. A. Knoll, Physics-based preconditioners for ocean simulation, SIAM J. Sci. Comput. 35 (2013) S445-S464.

[9] J. Thies, F. Wubs, H. Dijkstra, Bifurcation analysis of 3D ocean flows using a parallel fully-implicit ocean model, Ocean Model. 30 (2009) 287-297.

[10] W. Weijer, H. Dijkstra, H. Öksüzoğlu, F. Wubs, A. de Niet, A fully-implicit model of the global ocean circulation, J. Comput. Phys. 192 (2003) 452470.

[11] F. Wubs, A. de Niet, H. Dijkstra, The performance of implicit ocean models on B-and C-grids, J. Comput. Phys. 211 (2006) 210-228.

[12] K. Evans, D. Rouson, A. Salinger, M. Taylor, W. Weijer, J. White, A scalable and adaptable solution framework within components of the community climate system model, Computational Science-ICCS 2009 (2009) $332-341$. 
[13] D. A. Knoll, D. E. Keyes, Jacobian-free Newton-Krylov methods: a survey of approaches and applications, J. Comput. Phys. 193 (2004) 357-397.

[14] B. Nadiga, M. Taylor, J. Lorenz, Ocean modelling for climate studies: Eliminating short time scales in long-term, high-resolution studies of ocean circulation, Math. Comput. Modell. 44 (2006) 870-886.

[15] R. Smith, P. Gent, Reference maunal for the Parallel Ocean Program (POP), Technical Report Los Alamos Technical Report LA-UR-02-2484, Los Alamos National Laboratory, 2002.

[16] W. Weijer, Reference manual for the implicit version of the parallel ocean program (imPOP), Technical Report Los Alamos Technical Report LAUR-09-7040, Los Alamos National Laboratory, 2009.

[17] D. A. Knoll, V. A. Mousseau, L. Chacón, J. M. Reisner, Jacobian-free Newton-Krylov methods for the accurate time integration of stiff wave systems, J. Sci. Comp. 25 (2005) 213-230.

[18] H. Park, R. R. Nourgaliev, R. C. Martineau, D. A. Knoll, On physicsbased preconditioning of the Navier-Stokes equations, J. Comput. Phys. 228 (2010) 9131-9146.

[19] G. Chen, L. Chacón, D. C. Barnes, An energy-and charge-conserving, implicit, electrostatic particle-in-cell algorithm, J. Comput. Phys. 230 (2011) 7018-7036.

[20] W. Ying, D. J. Rose, C. S. Henriquez, Efficient fully implicit time integration methods for modeling cardiac dynamics, IEEE Trans. Biomed. Eng. 55 (2008) 2701-2711.

[21] S. Y. Kadioglu, D. A. Knoll, A fully second order implicit/explicit time integration technique for hydrodynamics plus nonlinear heat conduction problems, J. Comput. Phys. 229 (2010) 3237-3249.

[22] S. Y. Kadioglu, D. A. Knoll, R. B. Lowrie, R. M. Rauenzahn, A second order self-consistent IMEX method for radiation hydrodynamics, J. Comput. Phys. 229 (2010) 8313-8332.

[23] J.-F. Lemieux, D. A. Knoll, M. Losch, C. Girard, A second-order accurate in time implicit-explicit (IMEX) integration scheme for sea ice dynamics, J. Comput. Phys. (2014).

[24] G. Chen, L. Chacón, D. C. Barnes, An efficient mixed-precision, hybrid cpu-gpu implementation of a nonlinearly implicit one-dimensional particlein-cell algorithm, J. Comput. Phys. 231 (2012) 5374-5388.

[25] W. T. Taitano, D. A. Knoll, L. Chacón, G. Chen, Development of a consistent and stable fully implicit moment method for Vlasov-Ampère particle in cell (PIC) system, SIAM J. Sci. Comput. 35 (2013) S126-S149. 
[26] D. A. Knoll, H. Park, K. Smith, Application of the jacobian-free newtonkrylov method to nonlinear acceleration of transport source iteration in slab geometry, Nucl. Sci. Eng. 167 (2011) 122.

[27] H. Park, D. Knoll, C. Newman, Nonlinear acceleration of transport criticality problems, Nucl. Sci. Eng. 172 (2012) 52.

[28] H. Park, D. Knoll, R. Rauenzahn, C. Newman, J. Densmore, A. Wollaber, An efficient and time accurate, moment-based scale-bridging algorithm for thermal radiative transfer problems, SIAM J. Sci. Comput. 35 (2013) S18S41.

[29] H. Park, D. Knoll, R. Rauenzahn, A. Wollaber, J. Densmore, A consistent, moment-based, multiscale solution approach for thermal radiative transfer problems, Transp. Theory Stat. Phys. 41 (2012) 284-303.

[30] A. J. Wallcraft, E. P. Chassignet, H. E. Hurlburt, T. L. Townsend, $1 / 25^{\circ}$ Atlantic Ocean Simulation Using HYCOM, Technical Report No. NRL/PP/7320-05-5263, Naval Research Laboratory, Stennis Space Center, MS, Oceanography Div., 2005.

[31] K. Datta, S. Kamil, S. Williams, L. Oliker, J. Shalf, K. Yelick, Optimization and performance modeling of stencil computations on modern microprocessors, SIAM Rev. 51 (2009) 129-159.

[32] M. Wittmann, G. Hager, J. Treibig, G. Wellein, Leveraging shared caches for parallel temporal blocking of stencil codes on multicore processors and clusters, Parallel Process. Lett. 20 (2010) 359-376.

[33] D. Wonnacott, Using time skewing to eliminate idle time due to memory bandwidth and network limitations, in: Parallel and Distributed Processing Symposium, 2000. IPDPS 2000. Proceedings. 14th International, IEEE, 2000, pp. 171-180.

[34] C. Ding, Y. He, A ghost cell expansion method for reducing communications in solving PDE problems, in: Supercomputing, ACM/IEEE 2001 Conference, IEEE, 2001, pp. 55-55.

[35] J. Lambert, Numerical methods for ordinary differential systems: the initial value problem, John Wiley \& Sons, Inc., 1991.

[36] T. Ringler, M. Petersen, R. L. Higdon, D. Jacobsen, P. W. Jones, M. Maltrud, A multi-resolution approach to global ocean modeling, Ocean Model. 69 (2013) 211-232.

[37] R. Pacanowski, K. Dixon, A. Rosati, The GFDL modular ocean model users guide. GFDL Ocean Group Tech Rep 2, Technical Report, Geophysical Fluid Dynamics Laboratory, Princeton, USA, 1993. 
[38] C. T. Kelley, Iterative Methods for Linear and Nonlinear Equations, SIAM, Philadeplhia, PA, 1995.

[39] Y. Saad, Iterative Methods for Sparse Linear Systems, The PWS Series in Computer Science, PWS Publishing Company, Boston, MA, 1995.

[40] M. Pernice, H. F. Walker, NITSOL: a Newton iterative solver for nonlinear systems, SIAM J. Sci. Comp. 19 (1998) 302-318.

[41] P. N. Brown, Y. Saad, Hybrid Krylov methods for nonlinear systems of equations, SIAM J. Sci. Statist. Comput. 11 (1990) 450-481.

[42] R. Dembo, S. C. Eisenstat, T. Steihaug, Inexact Newton methods, SIAM J. Numer. Anal. 19 (1982) 400-408.

[43] S. C. Eisenstat, H. F. Walker, Choosing the forcing terms in a inexact Newton method, SIAM J. Sci. Comput. 17 (1996) 16-32.

[44] L. Chacón, D. A. Knoll, J. M. Finn, An implicit, nonlinear reduced resistive MHD solver, J. Comput. Phys. 178 (2002) 15-36.

[45] D. A. Knoll, W. J. Rider, A multigrid preconditioned Newton-Krylov method, SIAM J. Sci. Comput. 21 (2000) 691-710.

[46] U. Trottenberg, C. W. Oosterlee, A. Schüller, Multigrid, Academic Press, 2000.

[47] A. Arakawa, V. R. Lamb, Computational Design of the Basic Dynamical Processes of the UCLA General Circulation Model, in: J. Chang (Ed.), Methods in Computational Physics, volume 17, Academic Press, New York, 1977, pp. 173-265.

[48] R. Stallman, Using the GNU compiler collection, Free Software Foundation, Cambridge, MA (2012).

[49] E. Gabriel, G. E. Fagg, G. Bosilca, T. Angskun, J. J. Dongarra, J. M. Squyres, V. Sahay, P. Kambadur, B. Barrett, A. Lumsdaine, R. H. Castain, D. J. Daniel, R. L. Graham, T. S. Woodall, Open MPI: Goals, concept, and design of a next generation MPI implementation, in: Proceedings, 11th European PVM/MPI Users' Group Meeting, Budapest, Hungary, 2004, pp. 97-104.

[50] J. Li, W. K. Liao, A. Choudhary, R. Ross, R. Thakur, W. Gropp, R. Latham, A. Siegel, B. Gallagher, M. Zingale, Parallel netCDF: A scientific high-performance I/O interface, in: Proceedings of ACM/IEEE conference on Supercomputing, ACM Press, 2003, p. 39.

[51] M. A. Heroux, R. A. Bartlett, V. E. Howle, R. J. Hoekstra, J. J. Hu, T. G. Kolda, R. B. Lehoucq, K. R. Long, R. P. Pawlowski, E. T. Phipps, A. G. Salinger, H. K. Thornquist, R. S. Tuminaro, J. M. Willenbring, A. Williams, K. S. Stanley, An overview of the Trilinos project, ACM Trans. Math. Softw. 31 (2005) 397-423. 
[52] M. Gee, C. Siefert, J. Hu, R. Tuminaro, M. Sala, ML 5.0 Smoothed Aggregation User's Guide, Technical Report SAND2006-2649, Sandia National Laboratories, 2006.

[53] J. Marotzke, P. Welander, J. Willebrand, Instability and multiple steady states in a meridional-plane model of the thermohaline circulation, Tellus A 40 (1988) 162-172.

[54] J. Willert, W. T. Taitano, D. Knoll, Leveraging Anderson Acceleration for improved convergence of iterative solutions to transport systems, J. Comput. Phys. 273 (2014) 278-286. 